\title{
Evaluation of stiffness matrix in the static and dynamic elasticity problems
}

\author{
Eric $\mathrm{Li}^{1,2}, \mathrm{ZC} \mathrm{He}^{1,2,3^{*}}, \mathrm{GR} \mathrm{Liu}^{4}$ \\ ${ }^{1}$ State Key Laboratory of Advanced Design and Manufacturing for Vehicle Body, Hunan University, Changsha, \\ 410082 P. R. China \\ ${ }^{2}$ Department of Mechanical and Automation Engineering, The Chinese University of Hong Kong, Shatin, NT, Hong \\ Kong, China \\ ${ }^{3}$ State Key Laboratory of Structural Analysis for Industrial Equipment, Dalian University of Technology, Dalian \\ 116024, P.R.China \\ ${ }^{4}$ Department of Aerospace Engineering and Engineering Mechanics, University of Cincinnati, Cincinnati, OH \\ 45221-0070, USA
}

\begin{abstract}
A general evaluation technique (GET) for stiffness matrix in the finite element methods (FEM) using modified integration rule with alternate integration points $r \in[0,1]$ rather than the standard Gauss points is proposed. The GET is examined using quadrilateral elements for elasticity problems. For the first time, we have found that the desired softening and stiffening effect can be achieved with adjustments of integration point $r$. This allows the FEM model to achieve better accuracy, and handle special problems, such as hourglass instability and volumetric locking. Ideal regions for the integration point $r$ are found to overcome the hourglass and volumetric locking issues for the overestimation problems. In addition, the exact solution of FEM model with optimal $r$ value in terms of strain energy can be always obtained for general overestimation problems of elasticity. More importantly, the optimal integration point $r$ obtained from static case can be directly applied to dynamic problems to improve the transient displacement significantly. The intensive numerical examples including the static, dynamic, compressible and nearly incompressible material problems are analyzed to verify the accuracy and properties of GET. Furthermore, the implementation of GET is extremely simple without increasing computational cost.
\end{abstract}

Key words: Optimal integration points; Reduced integration; Hourglass; Zero Mode; Volumetric locking

\footnotetext{
1* State Key Laboratory of Advanced Design and Manufacturing for Vehicle Body, Hunan University, Changsha, 410082 P. R. China' Email address: hezhicheng815@hnu.edu.cn
} 


\section{Introduction}

In the industry, analytical solutions seldom exist in solving the partial differential equations (PDE) which govern the physical law of engineering problems. Therefore, many powerful numerical methods such as finite element method (FEM) [1-6], finite different method (FDM) [7], finite volume method (FVM) [8], boundary element method (BEM) [9] and meshfree method [10] have been proposed. These methods and techniques not only provide effective tools for many engineering problems, but also explore our minds in the quest for even more efficient and robust numerical methods.

Based on the well-known and well-established Hilbert space theory, the weak formulation of FEM with many commercial software packages available is probably the most popular numerical method used for general engineering problems [1-4]. In general, the lower-order quadrilateral elements in 2D and brick elements in 3D are very efficient in FEM, and such elements are widely used in engineering problems due to its accuracy and efficiency [11-13]. However, the FEM model discretized by quadrilateral elements using full integration behaves overly-stiff, leading to underestimation of strain energy. In addition, the locking issue of FEM model exists in nearly incompressible problems when the bulk modulus becomes infinite.

Reduced integration (RI) is widely accepted to be able to soften the overly-stiff FEM model [14-16]. However, zero energy modes or hour-glass phenomenon $[11,17,18]$ may arise in the low order of RI technique with singular stiffness matrix, which is the root cause of instability. Although this spurious singular mode caused by RI is cured by embedding the hourglass control methods [11], the artificial parameter in the hourglass control is very sensitive to the parameter and the aspect ratio of an element [17]. The selective reduced integration (SRI) developed by Hughes $[19,20]$ and Malkus [21] is very effective to overcome the spurious zero energy model, 
but the computational efficiency for SRI is worse than RI [17]. Another popular method proposed by Kosloff and Frazier is stabilization matrix with one point quadrature stiffness matrix [22].

On the other hand, a lot of research effort has been made in the past to deal with the volumetric locking issue for nearly incompressible materials. The selective integration is a common approach which decomposes the material properties matrix into two parts: the volumetric part and deviatoric part, and the reduced integration and full integration are used to compute the stiffness matrix of volumetric and deviatoric part respectively. However, the separation of material properties of matrix into volumetric and deviatoric part for anisotropic and/or nonlinear cases are not always available. The second popular remedy for volumetric locking is B-bar or F-bar projection method [20, 23, 24] for anisotropic and non-linear media. The key idea of this scheme is to use a stabilization matrix together with the reduced integrated matrix using reduced integration [12]. Compared with SI, the second approach is more effective for generalized elastic problems with nearly incompressible property. In other front of development, mixed formulations $[15,19,21,25-29]$ have been proposed to soften the overlystiff FEM model, which provides an effective way of overcoming the volumetric locking issues.

Alternatively, smoothed finite element method (SFEM) [30-34] developed by Liu et. al provides an effective way to soften the discretized model to overcome the drawback of reduced integration issues. Recently, Liu et al. [59] has formulated a node-based smoothed finite element using a mesh of polygonal elements. The NS-FEM is considered as overly-soft model that provides the upper bound of strain energy. In addition, NS-FEM is immune from volumetric locking issues. Furthermore, an alpha finite element method ( $\alpha$ FEM) [12] by scaling the gradient of strains and Jacobian matrices with a scaling factor $\alpha$ for quadrilateral elements is developed. 
The $\alpha$ FEM approach also works well for volumetric locking problems, by simply replacing the strain gradient matrix with a stabilization matrix. More importantly, the nearly exact or best possible solutions can be obtained with the adjustment of alpha value. Following this, a variationally consistent alpha finite element method (VC $\alpha \mathrm{FEM}$ ) [35] for quadrilateral elements is developed by constructing an assumed strain field, in which the gradient of the compatible strain field is scaled with a free parameter. The VC $\alpha$ FEM can produce both lower and upper bounds to the exact solution in the strain energy for all elasticity problems by choosing a proper $\alpha \in[0$, $\alpha_{\text {upper] }}$.

Motivated from the modified integration rules in the computation of the mass and stiffness for acoustic problems with quadrilateral mesh developed by Guddati [36, 37] and massredistributed (MR) method [38-41]to alter the mass matrix, this work develops a generalized formulation of stiffness using MIR with flexible integration points for elastic problems. With adjustment of integration points in the stiffness, the softening and stiffening effect in the FEM model can be altered, if so desired. For the first time, we have found that the integration points in the stiffness $r \in[0.001,0.01]$ is able to remove the hourglass instability, and the accuracy for $r \epsilon$ $[0.001,0.01]$ is much better than that using full integration point. In addition, the volumetric locking can be overcome by using integration points $r \in\left[(0.5-v),(0.5-v) e^{1}\right](v$ is the Poisson's ration for nearly incompressible materials). More importantly, the optimal integration point $r$ obtained from static case can be directly applied to dynamic problems to improve the transient displacement significantly. This is fantastic in the development of numerical method for general elastic problems. Furthermore, the implementation of the proposed formulation of stiffness is extremely easy without changing FEM code and increasing computational time. 
The outline of this paper is as follows: Section 2 outlines the general evaluation technique of stiffness using modified integration rule with different integration point; the strategies to overcome the volumetric and hourglass effect are presented in Section 3. In Section 4, intensive numerical examples are studied to verify the novel properties of GET in the static and dynamic models including compressible and incompressible materials. Finally, the conclusion is presented in Section 5.

\section{A general evaluation technique (GET) of stiffness with flexible integration}

\section{points}

\subsection{A brief of GET model in static and dynamic problems}

For more effective discussion, the standard formulation of FEM [4] is first briefed. In the FEM, the weak form with the arbitrariness of virtual nodal displacements can be always expressed in the following discretized algebraic equation:

$$
\mathbf{K u}=\mathbf{F}
$$

where

$$
\begin{gathered}
\mathbf{K}=\int_{\Omega} \mathbf{B}^{\mathrm{T}} \mathbf{D B} \mathrm{d} \Omega \\
\mathbf{F}=\int_{\Omega} \mathbf{N}_{I}^{\mathrm{T}}(\mathbf{x}) \mathbf{b} \mathrm{d} \Omega+\int_{\Gamma_{t}} \mathbf{N}_{I}^{\mathrm{T}}(\mathbf{x}) \overline{\mathbf{t}} \mathrm{d} \Gamma
\end{gathered}
$$

where $\mathbf{B}$ is the strain matrix, $\mathbf{N}$ is the shape function, $\mathbf{b}$ is the vector of body force, $\overline{\mathbf{t}}$ is the vector of the prescribed traction, and $\mathbf{D}$ is the matrix of material constants defined as follows:

$$
\mathbf{D}=\frac{E}{1-v^{2}}\left[\begin{array}{ccc}
1 & v & 0 \\
v & 1 & 0 \\
0 & 0 & \frac{1-v}{2}
\end{array}\right] \quad \text { Plane stress }
$$




$$
\mathbf{D}=\frac{E(1-v)}{(1+v)(1-2 v)}\left[\begin{array}{ccc}
1 & \frac{v}{1-v} & 0 \\
\frac{v}{1-v} & 1 & 0 \\
0 & 0 & \frac{1-2 v}{2(1-v)}
\end{array}\right] \quad \text { Plane strain }
$$

where $E$ is the Young's Modulus, and $v$ is the Poisson's ratio.

Two types of boundary conditions including essential/displacement and Neumann (or natural/stress) boundary conditions.

Essential boundary conditions is given as follows:

$$
u=u_{\Gamma} \quad \text { on } \Gamma_{u}
$$

where $u_{\Gamma}$ stands for the vector of the prescribed displacements on the essential boundary $\Gamma$.

Neumann boundary conditions is expressed:

$$
\mathbf{L}_{n}^{\mathrm{T}} \sigma=\overline{\mathbf{t}} \quad \text { on } \Gamma_{t}
$$

where $\mathbf{L}_{\mathrm{n}}$ is the matrix of unit outward normal that can be defined as follows:

$$
\mathbf{L}_{n}=\left[\begin{array}{cc}
n_{x} & 0 \\
0 & n_{y} \\
n_{y} & n_{x}
\end{array}\right] \quad \text { on } \Gamma_{t}
$$

in which $n_{x}$ and $n_{y}$ denote the unit outward normal in $x$ and $y$ directions respectively.

In numerical performance, the isoparametric elements and Gauss integration are usually used to calculate the entries of stiffness matrix $\mathbf{K}$ as follows 


$$
\begin{aligned}
\mathbf{K}_{I J} & =\int_{\Omega} \mathbf{B}_{\mathrm{I}}^{\mathrm{T}}(\mathbf{x}) \mathbf{D} \mathbf{B}_{J}(\mathbf{x}) \mathrm{d} \Omega \\
& =\int_{-1}^{1} \int_{-1}^{1} \mathbf{B}_{I}^{\mathrm{T}}(\mathbf{x}(\xi, \eta)) \mathbf{D} \mathbf{B}_{J}(\mathbf{x}(\xi, \eta))|\mathbf{J}(\xi, \eta)| \mathrm{d} \xi \mathrm{d} \eta \\
& =\sum_{i=1}^{N_{1}} \sum_{j=1}^{N_{2}} \mathbf{B}_{I}^{\mathrm{T}}\left(\mathbf{x}\left(\xi_{i}, \eta_{i}\right)\right) \mathbf{D} \mathbf{B}_{J} \times\left(\mathbf{x}\left(\xi_{i}, \eta_{i}\right)\right)\left|\mathbf{J}\left(\xi_{i}, \eta_{i}\right)\right| W_{i} \bar{W}_{j}
\end{aligned}
$$

where $N_{1}$ and $N_{2}$ are the number of Gauss integration points in the $\xi$ and $\eta$ axes, respectively. In addition, $\mathbf{J}$ is Jacobi matrix, $\xi_{i}$, and $\eta_{i}$ are the integration points, and $W_{i}$ and $W_{j}$ are weighting coefficients.

As shown in Fig. 1, two types of integration points are used: one is full integration point; the other is reduced integration. In the full integration,

$$
\begin{gathered}
\text { Point 1: }\left(-\frac{1}{\sqrt{3}}, \frac{1}{\sqrt{3}}\right) \text {, point } 2:\left(\frac{1}{\sqrt{3}}, \frac{1}{\sqrt{3}}\right) \\
\text { Point 3: }\left(-\frac{1}{\sqrt{3}},-\frac{1}{\sqrt{3}}\right) \text {, point } 4:\left(\frac{1}{\sqrt{3}},-\frac{1}{\sqrt{3}}\right)
\end{gathered}
$$

In the reduced integration method, only one integration point $(0,0)$ is employed to compute the stiffness.

In this work, the general formulation of stiffness using modified integration rule [36] with flexible integration points as shown in Fig. 2 is proposed:

$$
\begin{gathered}
\text { Point 1: }(-r, r), \text { point 2: }(r, r) \\
\text { Point 3: }(-r,-r) \text {, point 4: }(r,-r)
\end{gathered}
$$

where $r \in[0,1]$.

According to Eq. (11), when $r=0$, the GET model is identical with standard FEM using reduced integration. When $r=\frac{1}{\sqrt{3}} \approx 0.577$, the GET model is the same as standard FEM using full integration. 
Based on Eqs. (2)- (5) with consideration of damping effect, the governing equation for the dynamic elasticity problem can be written in the following matrix form:

$$
\mathbf{M u ̈}+\mathbf{c u}+\mathbf{K u}=\mathbf{F}
$$

where

$$
\begin{array}{ll}
\mathbf{M}=\rho \int_{\Omega} \mathbf{N}^{\mathrm{T}} \mathbf{N} \mathrm{d} \Omega & \text { The mass matrix } \\
\mathbf{c}=\alpha \mathbf{M}+\beta \mathbf{K} & \text { Damping matrix }
\end{array}
$$

where $\alpha$ and $\beta$ are the damping coefficients, $\rho$ is the density of the material.

The displacement could be determined using the following formula based on the central difference method [42]:

$$
\begin{gathered}
\dot{\mathbf{u}}=\frac{\mathbf{u}_{t+1}-\mathbf{u}_{t-1}}{2 \Delta t} \\
\ddot{\mathbf{u}}=\frac{\mathbf{u}_{t+1}-2 \mathbf{u}_{t}+\mathbf{u}_{t-1}}{\Delta t^{2}}
\end{gathered}
$$

The procedure to update the displacement is done in the following way. First, determine the acceleration

$$
\ddot{\mathbf{u}}^{t}=\mathbf{M}^{-1}\left(\mathbf{F}^{t}-\mathbf{c} \dot{\mathbf{u}}^{t}-\mathbf{K} \mathbf{u}^{t}\right)
$$

Then compute the velocity from the acceleration

$$
\dot{\mathbf{u}}^{t+0.5 \Delta t}=\dot{\mathbf{u}}^{t-0.5 \Delta t}+\Delta t \ddot{\mathbf{u}}^{t}
$$

Finally, the displacement can be obtained:

$$
\mathbf{u}^{t+\Delta t}=\mathbf{u}^{t}+\Delta t \dot{\mathbf{u}}^{t+0.5 \Delta t}
$$

\subsection{Properties of GET model}




\subsubsection{Classification of underestimation or overestimation problem}

It is very straightforward to determine whether a specific problem belongs to an overestimation or underestimation of the exact solution in the GET model. The simple procedure is illustrated as follows: using two coarse meshes with different densities to calculate the strain energy $E(r)$.

$$
\begin{gathered}
\left.E\right|_{\text {coarse mesh }}<\left.E\right|_{\text {fine mesh }} \rightarrow \text { underestimation problem } \\
\left.E(r)\right|_{\text {caarse mesh }}>\left.E(r)\right|_{\text {fine mesh }} \rightarrow \text { overstimation problem }
\end{gathered}
$$

\subsubsection{Lower bound property of strain energy}

The exact strain energy is defined as follows:

$$
E_{\text {exact }}=\left.\left.\frac{1}{2} \sum_{i=1}^{N_{e}} \int_{\Omega_{i}} \boldsymbol{\varepsilon}_{i}^{\mathrm{T}}\right|_{\text {exact }} \mathbf{D} \boldsymbol{\varepsilon}_{i}\right|_{\text {exact }} \mathrm{d} \Omega
$$

where $\varepsilon$ is the exact strain in each element.

In the GET model, the

$$
E_{G E T}=\frac{1}{2} \mathbf{u}^{\mathrm{T}} \mathbf{K}_{\mathrm{GET}} \mathbf{u}=\int_{-1}^{1} \int_{-1}^{1}(\hat{\boldsymbol{\varepsilon}}(r, \xi, \eta))^{\mathrm{T}} \mathbf{D} \hat{\boldsymbol{\varepsilon}}(r, \xi, \eta)|\mathbf{J}(r, \xi, \eta)| \mathrm{d} \xi \mathrm{d} \eta
$$

The strain $\hat{\boldsymbol{\varepsilon}}$ computed by GET model is expressed as follows:

$$
\hat{\boldsymbol{\varepsilon}}(\mathbf{x}(r, \xi, \eta))=\nabla_{s} \mathbf{u}^{h}=\sum_{I=1}^{N P} \mathbf{B}_{I}(\mathbf{x}(r, \xi, \eta)) \mathbf{u}_{I}
$$

In Eq. (24), the $\mathbf{B}\left(\mathbf{x}_{k}\right)$ is calculated as follows:

$$
\mathbf{B}_{I}\left(\mathbf{x}_{k}\right)=\left[\begin{array}{cc}
\frac{\partial \mathbf{N}_{I}(\mathbf{x})}{\partial x} & 0 \\
0 & \frac{\partial \mathbf{N}_{I}(\mathbf{x})}{\partial y} \\
\frac{\partial \mathbf{N}_{I}(\mathbf{x})}{\partial y} & \frac{\partial \mathbf{N}_{I}(\mathbf{x})}{\partial x}
\end{array}\right]
$$


As $r=1.0$, the GET becomes the stiffest model, which gives the minimum strain energy. Thus, The strain energy $E(r=1)$ is an underestimation of the exact strain energy.

$$
E_{G E T}(r=1)=\left.\frac{1}{2} \mathbf{u}^{\mathrm{T}} \mathbf{K}_{\mathrm{GET}} \mathbf{u}\right|_{r=1}<E_{G E T}\left(r=\frac{1}{\sqrt{3}}\right)=\left.\frac{1}{2} \mathbf{u}^{\mathrm{T}} \mathbf{K}_{\mathrm{GET}} \mathbf{u}\right|_{r=\frac{1}{\sqrt{3}}}<E_{\text {exact }}
$$

\subsubsection{Upper bound property of strain energy for overestimation problem}

When $r=0.0$, the GET becomes the standard FEM model using reduced integration. The strain energy $E(r=0)$ is an upper bound solution of the exact strain energy for overestimation problem.

$$
E_{G E T}(r=0)=\left.\frac{1}{2} \mathbf{u}^{\mathrm{T}} \mathbf{K}_{\mathrm{GET}} \mathbf{u}\right|_{r=0}>E_{\text {exact }}
$$

\subsubsection{Solution continuity for displacement and strain energy}

As $r$ varies from 0.0 to 1.0, the solutions of the GET model are continuous functions from the solutions of the FEM using full and reduced integration models.

\subsubsection{The exact $r$ solution in dynamic problem}

The properties of GET model (2.2.1-2.2.4) indicate that $r \in[0,1]$, the strain energy of the GET model gives the biggest value at $r=0.0$, but achieves the smallest value at $r=1.0$, and we can produce the exact solution of strain energy using $0.0 \leq r<1.0$ in the GET model for static problem. Based on the optimal $r_{\text {exact }}$ value corresponding to the exact solution in static problem, the 2D numerical results of GET model in dynamic problem using $r_{\text {exact }}$ can been improved significantly. This is extremely important in the simulation of dynamic problems, which is demonstrated in section 4.2 . 


\section{Strategies to overcome hourglass and volumetric locking}

Depending on the boundary condition, the numerical results of reduced integration are either "overestimation" or "underestimation" of the exact solution [12, 35]. It is well known that the

numerical instability such as hourglass and volumetric locking using reduced integration may occur for overestimation problems $[12,35,43]$. In this section, the strategies to overcome the hourglass and volumetric locking issue are analyzed in detail with simple adjustment of integration point for overestimation problems.

\subsection{Small $r$ value in the treatment of hourglass effect}

First, a free vibration analysis using a free distorted quadrilateral element with a total of eight degrees of freedom (DOFs) as shown in Fig. 3 is conducted. The eigenvalues for different integration points are presented in Table 1. It is observed that there are three zero eigenvalues corresponding to three rigid movements of the element for all $r$ value. However, the $r=0$ corresponding to reduced integration gives two additional zero-energy modes which are the two "hour-glass" modes. From Table 1, it is seen that no "spurious" modes exist as long as $r>0$, which validates that the GET is always stable as $0.0<r \leqslant 1.0$.

The condition number of stiffness $\mathbf{K}$ for different integration point $r$ in the GET model is shown in Fig. 4 as $r>10^{-5}$. It is noted that the condition numbers are the same level for all integration points $r$. On the contrary, the integration point $r=0$ gives the infinite value of condition number. In order to ensure the stable solutions with optimal $r$ value in the GET model, the following three conditions must be satisfied [12]: first, two additional non-zero eigenvalues corresponding to spurious zero eigenvalues at $r=0$ are larger than 1, and these two additional non-zero eigenvalues should be small as possible compared to the others of non-zero eigenvalues; second, the condition number of matrix $\mathbf{K}$ computed by GET and full integration $(r=1 / \sqrt{3})$ should 
be comparable; third, three non-zero $6^{\text {th }}, 7^{\text {th }}, 8^{\text {th }}$ eigenvalues obtained from full integration corresponding to $r=1 / \sqrt{3}$ and GET should be the same. As long as these three conditions are satisfied [12], the stiffness matrix $\mathbf{K}$ has a good condition number to ensure numerical stability, and the numerical solutions are almost the same as those of the FEM using the reduced integration. Following this principle, it is found that the integration points $r \in[0.001,0.01]$ in this range are the best values to satisfy these three conditions.

As shown in Fig. 5, the vibration analysis with four fixed DOFs using one single quadrilateral element is conducted to study the property of GET further. The results of the condition number of matrix $\mathbf{K}$ are given in Fig. 6 as $r>0$. Again, the condition number $\mathbf{K}$ for the reduced integration $r=0$ becomes infinite. It is also noted that all the condition numbers for $r>0$ are comparable to that using FEM with full integration $(r=1 / \sqrt{ } 3)$.

Table 2 shows the eigenvalues computed by different integration points. Based on three conditions mentioned above, it is found that the range for $r \in[0.001,0.01]$ is the most suitable value to remove the spurious zero-energy mode, but it gives the same accuracy of reduced integration. As shown in Table 2, it is found that these two additional non-zero eigenvalues obtained from $r \in[0.001,0.01]$ are larger than 1.0 and very small compared to the $6^{\text {th }}, 7^{\text {th }}$ and $8^{\text {th }}$ nonzero eigenvalues.

The condition numbers for the stiffness $\mathbf{K}$ using various integration points are plotted in Fig. 6 as $r>0$. As shown in Fig. 6, all the condition numbers of the stiffness matrix $\mathbf{K}$ for any $r>0$ are comparable to that at $r=1 / \sqrt{ } 3$ corresponding to the standard Gauss integration.

\subsection{Small $r$ value in the treatment of volumetric locking issue}

The small $r \in[0.001,0.01]$ is only a good strategy to overcome the hourglass or zero mode for compressible materials. However, the standard FEM suffers the volumetric locking issue as the 
Poisson's ratio of material is close to 0.5 due to the overly-stiff property. Although the reduced integration of the standard FEM is a possible way to overcome such locking in the underestimation problems, the zero-energy modes or numerical instability still occur in the overestimation problems for nearly incompressible materials. In this Section, the eigenvalue for free vibration is investigated to select the optimal $r$ value to overcome the volumetric locking issue of incompressible materials.

Tables 3, 4, 5, 6 and 7 present the eigenvalues using different integration points in the GET model for the Poisson's ratio $v=0.499,0.4995,0.49995,0.499995$ and 0.4999995 . Again, two additional zero eigenvalues appear using reduced integration corresponding to $r=0$, which is the root cause for the numerical instability. It is observed that the $r \in\left[(0.5-v),(0.5-v) e^{1}\right]$ always ensures that the eigenvalues satisfy the three conditions mentioned above.

Table 8 describes the condition number for different integration points as the Poisson's ratio varies from 0.499 to 0.4999995 . It is expected that the $r=0$ for the reduced integration model gives the infinite value of condition number. On the other hand, all the condition numbers are comparable as $r>0$. This has confirmed that the proposed formulation of small $r$ value $\epsilon[(0.5-v)$, $\left.(0.5-v) e^{1}\right]$ is very effective to alleviate the volumetric locking issue for nearly incompressible materials.

\section{Numerical examples}

In order to demonstrate the properties of the present GET model, several numerical examples are analyzed including static and dynamic elastic problems with compressible and nearly incompressible materials. In order to evaluate the numerical results in a quantitative way, the following error indicator for the displacement is defined: 


$$
e_{d}=\sqrt{\frac{\sum_{i=1}^{N_{n}}\left(u_{i}^{e x}-u_{i}^{n u m}\right)^{2}}{\sum_{i=1}^{n}\left(u_{i}^{e x}\right)^{2}}}
$$

where the superscript $e x$ stands for the exact or analytical solution, num denotes the numerical solution obtained using GET.

The numerical strain energy $E_{n u m}$ is calculated by the following way:

$$
E_{\text {num }}=\frac{1}{2} \int_{\Omega}\left(\boldsymbol{\varepsilon}^{\text {num }}\right)^{\mathrm{T}} \mathbf{D}\left(\boldsymbol{\varepsilon}^{\text {num }}\right) d \Omega \quad \text { or } \quad E_{\text {num }}=\frac{1}{2} \mathbf{u}^{\mathrm{T}} \mathbf{K} \mathbf{u}
$$

where $\boldsymbol{\varepsilon}^{\text {num }}$ is the numerical solution of strain.

It is noted that GET model corresponding to $r=0$ is exactly the same as FEM using reduced

integration. The $r=\frac{1}{\sqrt{3}} \approx 0.577$ in the GET model becomes the identical formulation of the standard FEM using full integration. In all numerical examples, all the numerical solutions from GET model have been compared with standard FEM either reduced or full integration.

\subsection{Patch test}

It is necessary to conduct the patch test to ensure the convergence in the development of a numerical method for elastic problems [5]. Therefore, the first example is the standard patch test using the GET. A square domain with the dimension of $1 \times 1$ is studied, and the following displacements are prescribed on all the boundaries.

$$
\left\{\begin{array}{l}
u_{x}=0.6 x \\
u_{y}=0.6 y
\end{array}\right.
$$


The analytical solution for this patch test is a linear displacement field given by the above equation over the entire patch. Two sets of mesh including 121 regular and irregular mesh as shown in Fig. 7 are employed to conduct the patch test, and the displacement error as defined in Eq. (29) is computed. It is found that the displacement errors in the GET model for any $r \in[0,1]$ are found to be less than $1.0 \times 10^{-14}$ as shown in Fig. 8, which reaches almost the level of the machine precision. This example has strongly validated that the GET can pass the standard path test, which at least guarantees the linearly conforming.

\subsection{Cook's membrane}

The well-known Cook's membrane problem subjected to an in-plane distributed tip load $\mathrm{F}=$ 1, is shown in Fig. 9. The properties of the Cook's membrane are taken as follows: the Young's modulus is $E=1$, and the Poisson's ratio $v=1 / 3$. The mechanical boundary condition is depicted in Fig. 9. As the analytical solution for this Cook's membrane is not available, the reference values of the strain energy and the vertical displacement at center tip section are 12.015 [44] and 23.9642 [13], respectively.

Figure 10 shows the numerical solution of strain energy using different integration points in the GET model. Four sets of mesh using quadrilateral elements are employed in this study: $2 \times 2$, $4 \times 4,8 \times 8$ and $16 \times 16$. It is clearly observed that the upper and lower bound solutions of strain energy are obtained in the discretized model. The numerical solutions of GET approach the exact solution with increasing the number of node. More importantly, the numerical solutions of strain energy intercept with the exact solution as $r=0.16$.

Figure 11 describes the numerical results of the tip displacement in GET model using the different sets of mesh. Again, it is still found that the exact solution of displacement is obtained as the integration point $r$ is shifted from 0 to 1 . This example has revealed a key issue in the 
development of efficient numerical methods: the accuracy of numerical solutions of FEM can be improved significantly with the adjustment of integration point in the stiffness. This is a very important property, which implies that we can always obtain ultra-accurate solution with optimized $r$ value using quadrilateral element for overestimation problems.

The convergence rates for the strain energy and displacement are plotted in Figs. 12 and 13 respectively. Four typical integration points $r=1$ (the stiffest model), $r=0$ (standard FEM using reduced integration), $r=0.577$ (standard FEM using full integration) and $r=0.16$ (corresponding to nearly exact strain energy) are selected to investigate the property of GET model. As outlined in Figs. 12 and 13, the solutions of all these methods converge the exact solution with reducing the nodal spacing. In terms of accuracy, $r=0.16$ in the GET model gives the best results compared with the standard FEM using full integration and reduced integration. Again, the $r=1$ in the GET model behaves overly-stiff effect that produces the worst solutions.

In order to further demonstrate the advantages of the GET model, the dynamic solutions for different integration points are investigated in Fig. 14. In this example, the following parameters are chosen: density $\rho=1, \alpha=0.01$, and $\beta=0.2$ (the force, boundary conditions and other parameters are the same as static case). As shown in Fig. 14, it is noticed that the transient solutions from $r=0.16$ using coarse mesh $4 \times 4$ mesh agree very well with those with fine mesh $(32 \times 32)$ using full integration for dynamic problem. The numerical solutions from standard FEM either full or reduced integration are less accurate compared with GET model using $r=0.16$. This indicates that the optimal $r$ value obtained from the static case is also very effective to solve the transient problems. 


\subsection{Cantilever beam under a tip load}

A 2D cantilever beam with height $D=12 \mathrm{~m}$ and length $L=48 \mathrm{~m}$ is furthered analyzed to investigate the property of GET as shown in Fig. 15. The cantilever beam is subjected to a parabolic traction $\mathrm{P}=1000 \mathrm{~N}$ at free edge, and the material properties are taken as $E=30 \mathrm{MPa}$, and the Poisson's ratio $v=0.3$. It is noted that the displacement of the left hand side is constrained based on the computation of Eqs. (23) and (24). The exact solution of strain energy for this cantilever is 4.4746 [45].

$$
\begin{gathered}
u_{x}=\left[x(6 L-3 x)+(2+v)\left(y^{2}-\frac{D^{2}}{4}\right)\right] \frac{P y}{6 E I} \\
u_{y}=\left[x^{2}(3 L-x)+(4+5 v) \frac{D^{2} x}{4}+(L-x) 3 v y^{2}\right] \frac{-P y}{6 E I}
\end{gathered}
$$

The stresses corresponding to the displacement are given as follows:

$$
\sigma_{x x}(x, y)=\frac{y(L-x) P}{I} ; \quad \tau_{y y}(x, y)=-\frac{P}{2 I}\left(\frac{D^{2}}{4}-y^{2}\right) \quad \sigma_{y y}(x, y)=0 ;
$$

where $I$ is the moment of inertia for a beam that is defined by $\mathrm{D}^{3} / 12$.

The strain energy using different integration point $r$ in the GET model together with the exact solution is plotted in Fig. 16. Four sets of mesh using quadrilateral elements (52, 175, 637, 2425 nodes) are employed to validate the property of GET. Again, it is seen that the upper and lower bound of strain energy have been obtained. As $r<0.46$, the GET behaves an overly-soft model that gives the upper bound solutions. On the contrary, the GET produces an overly-stiff model with lower bound solutions as $r>0.46$. The displacement error of GET model is shown in Fig. 17. It is easily noticed that the integration point $r=0.47$ gives the minimum error of displacement. In addition, with increasing the number of elements, the displacement error 
reduces in the GET mode regardless of integration point $r$. This example has confirmed again that the GET has a property to provide the exact solution in terms of strain energy for overestimation problem.

To evaluate the influence of the mesh irregularities on the accuracy, this cantilever beam discretized by irregular mesh is tested. A shown in Fig. 18, two sets of irregular mesh with 52 and 175 nodes respectively are employed. The numerical results for the strain energy and displacement error are plotted in Fig. 19 using different sets of irregular mesh. As expected, both coarse and fine mesh models using irregular element are able to provide the exact solution of strain energy as $r=0.4$ shown in Fig. 19(a). This could be very important in the real application of engineering problems using irregular mesh to achieve ultra-accurate solutions. On the other hand, the $r=0.42$ in the GET model predicts the best solution of displacement as outlined in Fig. 19(b).

The dynamic performance of GET is evaluated again for this cantilever beam. In the dynamic analysis, the left hand side of beam is fixed. The Young's modulus $E=300 \mathrm{MPa}$, density $\rho=1000$ $\mathrm{kg} / \mathrm{m}^{3}$, and the uniform pressure $1000 \mathrm{~N} / \mathrm{m}$ is applied to upper edge (the remaining parameters are the same as those applied in the static case). First, we examine the optimal integration point in the static case, and two types of coarse mesh are used including 52 and 175 nodes. As shown in Fig. 20, the strain energy and vertical displacement (point A) versus flexible integration point $r$ are presented. As there is no analytical solution for this static case, the reference model with very fine quadrilateral elements (19601 nodes) is used to verify the optimal integration point. It is easy to see that the optimal integration point $r$ is equal to 0.44 in terms of strain energy and displacement solution.

Next, the vertical displacement at point A versus time is plotted in Fig. 21. In this dynamic example, the following damping parameters are employed: $\alpha=0.9, \beta=0.1$. It is obviously noticed 
that the optimal integration point $r=0.44$ obtained from static case still gives the best solutions in the dynamic model compared with $r=0$ (reduced integration of FEM model) and $r=0.577$ (full integration of FEM model). This is very significant in the simulation of dynamic model.

\subsection{Cylindrical pipe subjected to an inner pressure}

Figure 22(a) shows the 2D Lame problem subjected to internal pressure $P=6 \mathrm{kPa}$. The inner and outer radius of the sphere are $a=0.1 \mathrm{~m}, b=0.2 \mathrm{~m}$, respectively. The Young's modulus and Poisson's ratio for this cylinder are $E=21 \mathrm{MPa}$ and $v=0.3$. As the problem is axisymmetric, only one quarter of the cylinder is modeled as shown in Fig. 22(b). The analytical solution is available in polar coordinate system [1] for this benchmark problem.

$$
u_{l}=\frac{P a^{2} l(1+v)}{E\left(b^{2}-a^{2}\right)}\left[(1-2 v)+(1+v) \frac{b^{2}}{l^{2}}\right]
$$

where $l$ is the radial distance from the centroid of the sphere to the point of interest in the sphere.

The exact solutions for the stress can be expressed as follows:

$$
\sigma_{l}(l)=\left(1-\frac{b^{2}}{l^{2}}\right) \frac{a^{2} p}{b^{2}-a^{2}} \quad \sigma_{l \varphi}=0 \quad \sigma_{\varphi}(l)=\left(1+\frac{b^{2}}{l^{2}}\right) \frac{a^{2} p}{b^{2}-a^{2}}
$$

where $(l, \varphi)$ are the polar coordinates and $\varphi$ is measured counter-clockwise from the positive $x$ axis.

The strain energy for different integration point $r$ is plotted in Fig. 23. Three sets of mesh using quadrilateral element are adopted: $4 \times 8,8 \times 16$ and $16 \times 32$. It is seen that GET can bound the exact solutions from the blow and above using different integration points. This implies that the stiffening or softening properties of discretized model are altered by integration point $r$ in the GET model. As $r<0.42$, the GET model that behaves the overly-soft effect provides the upper bound solution, while the stiffening effect of the GET model is becoming stronger and 
stronger as $r>0.42$. It is also noted that the strain energy of numerical solution approaches the exact solution as the mesh density increases. In addition, it is found that the exact solution of strain energy can be obtained for these three sets of mesh. The $r=0.577$ corresponding to full integration of FEM model always provides a lower bound solution for these three sets of discretized models.

The displacement contour along $x$ direction using different integration point is presented in Fig. 24. It is obviously noticed that the reduced integration of FEM model corresponding to $r=0$ suffers the hourglass effect. On the other hand, the displacement contours using integration point $r=0.01$ and $r=0.001$ agree very well with the analytical solution, which validates that the small $r$ value $r \in[0.001,0.01]$ conducted in the analysis of eigenvalue for free vibration is very effective to alleviate the hourglass effect.

The displacement error versus the integration point $r$ in GET model is plotted in Fig. 25 as $r \geq 0.001$. Three different discretized models including $4 \times 8,8 \times 16$ and $16 \times 32$ quadrilateral elements are used. Again, it is noticed that displacement error is strongly related to the integration point $r$. As the $r=0$ corresponding to reduced integration of FEM model suffers the hourglass effect, the displacement error for this case is not shown in Fig. 25. It is noted that the displacement errors reduce in the GET model with increasing the number of nodes.

\subsection{Volumetric locking}

The volumetric locking issue with the same mechanical properties in Section 4.4 except the Poisson's ratio is then studied to study the performance of GET mode for nearly incompressible materials. Fig. 26 shows the displacement error $(4 \times 8$ quadrilateral elements $)$ using different integration points of GET model $(r>0)$ as the Poisson's ratio is changed from 0.499 to 0.499995 . It is expected that the integration point $r$ in the stiffness has a strong effect on the accuracy of 
displacement. The errors increase dramatically as the integration point $r$ increases from 0 to 1 . This is because the stiff property of discretized model is proportional to integration point $r$. Therefore, the deviation between the numerical solutions and the exact one is becoming larger and larger. Apparently, the integration point $r=1$ corresponding to the stiffest model gives the worst solution. As expected, the numerical results using the standard full integration of FEM model $(r=0.577)$ are still not desirable.

The prediction of displacement contour along $x$ direction using reduced integration of FEM model ( $r=0$ in the GET model) with $4 \times 8$ quadrilateral elements is depicted in Fig. 27 as the Poisson's ratio ranges $0.499,0.4995,0.49995$ and 0.499995 . The numerical results of displacement contours clearly indicate the reduced integration of FEM model suffers the hourglass effect severely.

The displacement error using $4 \times 8$ quadrilateral elements is shown in Fig. 28 for nearly incompressible materials. The Poisson's ratio ranging from $0.499,0.4995,0.49995,0.499995$ and 0.4999995 is studied to verify the proposed stable range of $r \in\left[(0.5-v),(0.5-v) e^{1}\right]$ value of integration point. It is observed that the integration point $r$ based on this formulation gives very good results. The displacement error is always limited to 0.0001 even the Poisson's ratio is very close to 0.5, which gives much smaller errors compared with standard Gauss integration approach.

Figures 29(a) and (b) plot the convergence rate of displacement norm errors using different integration points as the Poisson's ratio is equal to 0.499 and 0.499995 . Four typical integration points are studied: $r=1$ (the stiffest model), $r=0.577$ (full integration of FEM model), $r=0.5-v$ and $r=(0.5-v) e^{1}$. From Fig. 27, it is clearly observed that the convergence rate for both $r=0.5-v$ and $r=(0.5-v) e^{1}$ models give much more accurate solutions compared with $r=1$ and $r=0.577$. As the 
Poisson's ratio is approaching to 0.5 , the numerical solutions for $r=1$ and $r=0.577$ model (corresponding to FEM model using full integration) do not converge the exact solution as the number of elements increases. This is because the GET model for $r=1$ and $r=0.577$ suffers the volumetric locking issue.

\section{Conclusion}

For the first time, a general evaluation technique (GET) for stiffness matrix using flexible integration points is developed and discussed in detail for general overestimation of elasticity problems. Based on the theoretical analysis and numerical results, several conclusions can be made as follows:

a) The integration point $r$ in the stiffness plays an important role to determine the softening or stiffening properties of the discretized model.

b) The GET model always produces the lower and upper bounds to the exact solution in energy norm for overestimation problems.

c) The hourglass effect can be removed by using small $r$ value $r \in[0.001,0.01]$.

d) The integration point $r \in\left[(0.5-v),(0.5-v) e^{1}\right]$ in the GET model is able to overcome the volumetric locking issue.

e) The accuracy of displacement in static and dynamic problems is improved significantly using exact solution of strain energy.

f) The implementation of GET is extremely easy without changing FEM code. 


\section{Acknowledgements}

The project is supported by the Project funded by China Postdoctoral Science Foundation. The authors also wish to thank Research Project of the Science Fund of State Key Laboratory of Advanced Design and Manufacturing for Vehicle Body (Grant No. 51375001 and 31615002), Research Project of State Key Laboratory of Mechanical Systems and Vibration (MSV201711), and Research Project of State Key Laboratory of Structural Analysis for Industrial Equipment (Grant No. GZ 1609) for the support.

\section{Reference}

[1] T.J.R. Hughes, The finite element method : linear static and dynamic finite element analysis, Prentice Hall, Englewood Cliffs, N.J., 1987.

[2] J.N. Reddy, An introduction to the finite element method, 3rd ed., McGraw-Hill Higher Education, Boston ; New York, NY, 2006.

[3] O.C. Zienkiewicz, R.L. Taylor, D. Fox, The finite element method for solid and structural mechanics, seventh edition, Butterworth-Heinemann,, Oxford ; Waltham, Mass., 2014.

[4] G.R. Liu, S.S. Quek, The finite element method : a practical course, Second edition. ed.2013.

[5] C. Bagni, H. Askes, E.C. Aifantis, Gradient-enriched finite element methodology for axisymmetric problems, Acta Mechanica, 228 (2017) 1423-1444.

[6] A. Entezari, M.A. Kouchakzadeh, E. Carrera, M. Filippi, A refined finite element method for stress analysis of rotors and rotating disks with variable thickness, Acta Mechanica, 228 (2017) 575-594.

[7] D.J. Duffy, Finite difference methods in financial engineering : a partial differential equation approach, John Wiley, Chichester, England ; Hoboken, NJ, 2006.

[8] R.J. LeVeque, ebrary Inc., Finite volume methods for hyperbolic problems, Cambridge texts in applied mathematics, Cambridge University Press,, Cambridge ; New York, 2002, pp. xix, 558 p.

[9] L.C. Wrobel, M.H. Aliabadi, The boundary element method, J. Wiley, Chichester ; New York, 2002.

[10] G.R. Liu, Meshfree methods moving beyond the finite element method, CRC Press,, Boca Raton, Fla., 2010, pp. xxi, 770 p. 
[11] D.P. Flanagan, T. Belytschko, A Uniform Strain Hexahedron and Quadrilateral with Orthogonal Hourglass Control, Int J Numer Meth Eng, 17 (1981) 679-706.

[12] G.R. Liu, T. Nguyen-Thoi, K.Y. Lam, A novel FEM by scaling the gradient of strains with factor alpha (alpha FEM), Comput Mech, 43 (2009) 369-391.

[13] M. Fredriksson, N.S. Ottosen, Fast and accurate 4-node quadrilateral, Int J Numer Meth Eng, 61 (2004) 1809-1834.

[14] R.S. Sandhu, K.J. Singh, Reduced integration for improved accuracy of finite element approximations, Comput Method Appl M, 14 (1978) 23-37.

[15] S. Doll, K. Schweizerhof, R. Hauptmann, C. Freischlager, On volumetric locking of loworder solid and solid-shell elements for finite elastoviscoplastic deformations and selective reduced integration, Engineering Computations, 17 (2000) 874-902.

[16] O.C. Zienkiewicz, R.L. Taylor, J.M. Too, Reduced integration technique in general analysis of plates and shells, Int J Numer Meth Eng, 3 (1971) 275-290.

[17] B.C. Koh, N. Kikuchi, New Improved Hourglass Control for Bilinear and Trilinear Elements in Anisotropic Linear Elasticity, Comput Method Appl M, 65 (1987) 1-46.

[18] A. Cangiani, G. Manzini, A. Russo, N. Sukumar, Hourglass stabilization and the virtual element method, Int J Numer Meth Eng, 102 (2015) 404-436.

[19] T.J.R. Hughes, Equivalence of Finite-Elements for Nearly Imcompressible Elasticity, J Appl Mech-T Asme, 44 (1977) 181-183.

[20] T.J.R. Hughes, Generalization of Selective Integration Procedures to Anisotropic and NonLinear Media, Int J Numer Meth Eng, 15 (1980) 1413-1418.

[21] D.S. Malkus, T.J.R. Hughes, Mixed Finite-Element Methods - Reduced and Selective Integration Techniques - Unification of Concepts, Comput Method Appl M, 15 (1978) 63-81.

[22] D. Kosloff, G.A. Frazier, Treatment of Hourglass Patterns in Low Order Finite-Element Codes, Int J Numer Anal Met, 2 (1978) 57-72.

[23] A. Ortiz-Bernardin, J.S. Hale, C.J. Cyron, Volume-averaged nodal projection method for nearly-incompressible elasticity using meshfree and bubble basis functions, Comput Method Appl M, 285 (2015) 427-451.

[24] S.P. Oberrecht, J. Novak, P. Krysl, B-bar FEMs for anisotropic elasticity, Int J Numer Meth Eng, 98 (2014) 92-104. 
[25] L.R. Herrmann, Elasticity Equations for Incompressible and Nearly Incompressible Materials by a Variational Theorem, Aiaa J, 3 (1965) 1896-\&.

[26] F. Brezzi, M. Fortin, Mixed and Hybrid Finite Element Methods, Springer Series in Computational Mathematics,, Springer New York,, New York, NY, 1991, pp. 1 online resource.

[27] U. Heisserer, S. Hartmann, A. Duester, Z. Yosibash, On volumetric locking-free behaviour of p-version finite elements under finite deformations, Communications in Numerical Methods in Engineering, 24 (2008) 1019-1032.

[28] B.P. Lamichhane, A mixed finite element method for nearly incompressible elasticity and Stokes equations using primal and dual meshes with quadrilateral and hexahedral grids, J Comput Appl Math, 260 (2014) 356-363.

[29] M. Cervera, M. Chiumenti, Q. Valverde, C.A. de Saracibar, Mixed linear/linear simplicial elements for incompressible elasticity and plasticity, Comput Method Appl M, 192 (2003) 52495263.

[30] G.R. Liu, T.T. Nguyen, Smoothed finite element methods, Taylor \& Francis,, Boca Raton, 2010, pp. 691 p.

[31] E. Li, Z.C. He, X. Xu, G.R. Liu, Hybrid smoothed finite element method for acoustic problems, Comput Method Appl M, 283 (2015) 664-688.

[32] E. Li, Z.C. He, X. Xu, G.R. Liu, Y.T. Gu, A three-dimensional hybrid smoothed finite element method (H-SFEM) for nonlinear solid mechanics problems, Acta Mechanica, 226 (2015) 4223-4245.

[33] E. Li, Z. Zhang, C.C. Chang, G.R. Liu, Q. Li, Numerical homogenization for incompressible materials using selective smoothed finite element method, Compos Struct, 123 (2015) 216-232.

[34] T. Chau-Dinh, Q. Nguyen-Duy, H. Nguyen-Xuan, Improvement on MITC3 plate finite element using edge-based strain smoothing enhancement for plate analysis, Acta Mechanica, (2017) 1-23.

[35] G.R. Liu, H. Nguyen-Xuan, T. Nguyen-Thoi, A variationally consistent alpha FEM (VC alpha FEM) for solution bounds and nearly exact solution to solid mechanics problems using quadrilateral elements, Int J Numer Meth Eng, 85 (2011) 461-497.

[36] M.N. Guddati, B. Yue, Modified integration rules for reducing dispersion error in finite element methods, Comput Method Appl M, 193 (2004) 275-287. 
[37] B. Yue, M.N. Guddati, Dispersion-reducing finite elements for transient acoustics, J Acoust Soc Am, 118 (2005) 2132-2141.

[38] Z. He, G. Li, G. Zhang, G.-R. Liu, Y. Gu, E. Li, Acoustic analysis using a massredistributed smoothed finite element method with quadrilateral mesh, Engineering Computations, 32 (2015) 2292-2317.

[39] E. Li, Z.C. He, Y. Jiang, B. Li, 3D mass-redistributed finite element method in structuralacoustic interaction problems, Acta Mechanica, 227 (2016) 857-879.

[40] E. Li, Z.C. He, X. Xu, G.Y. Zhang, Y. Jiang, A faster and accurate explicit algorithm for quasi-harmonic dynamic problems, Int J Numer Meth Eng, 108 (2016) 839-864.

[41] E. Li, Z.C. He, Z. Zhang, G.R. Liu, Q. Li, Stability analysis of generalized mass formulation in dynamic heat transfer, Numerical Heat Transfer, Part B: Fundamentals, 69 (2016) 287-311.

[42] G. Noh, K.J. Bathe, An explicit time integration scheme for the analysis of wave propagations, Comput Struct, 129 (2013) 178-193.

[43] O.C. Zienkiewicz, R.L. Taylor, The finite element method for solid and structural mechanics, Elsevier Butterworth-Heinemann,, Amsterdam ; Boston, 2005, pp. xv, 631 p.

[44] D. Mijuca, M. Berkovic, On the efficiency of the primal-mixed finite element scheme, Advances in Computational Structural Mechanics, (1998) 61-68.

[45] S. Timoshenko, J.N. Goodier, Theory of elasticity, 3d ed., McGraw-Hill, New York, 1969. 


\section{Figure}

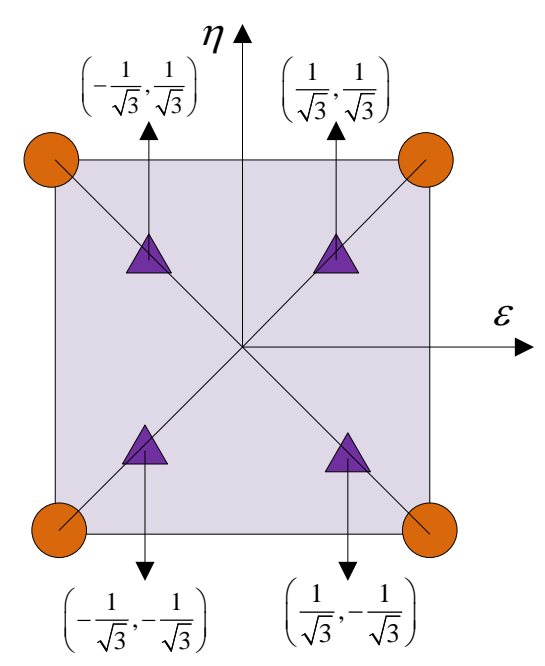

a) Full integration

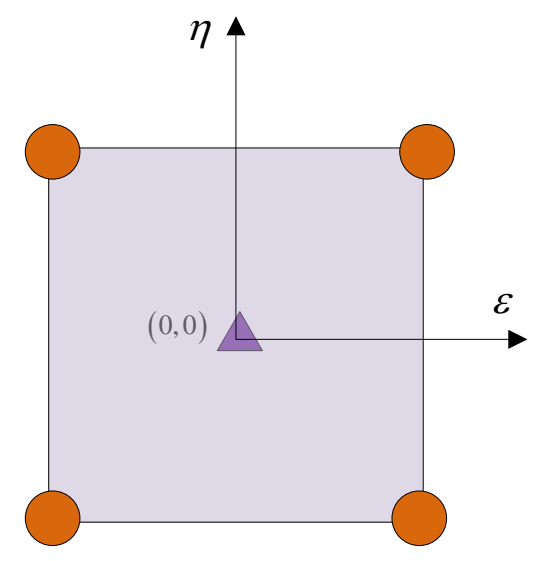

b) Reduced integration

Nodes of field Integration point

Figure 1: Gauss integration point 


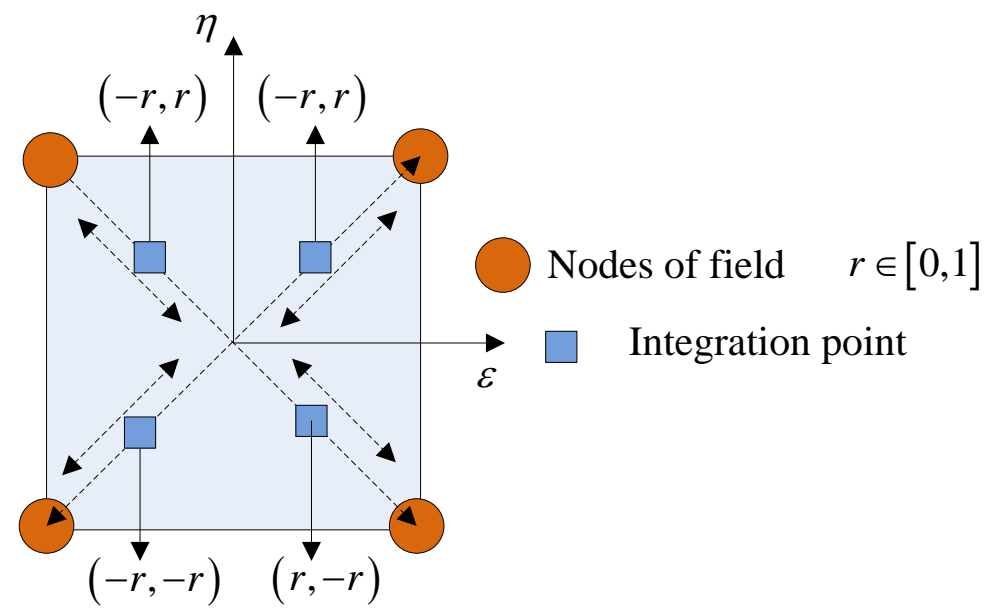

Figure 2: General integration point 


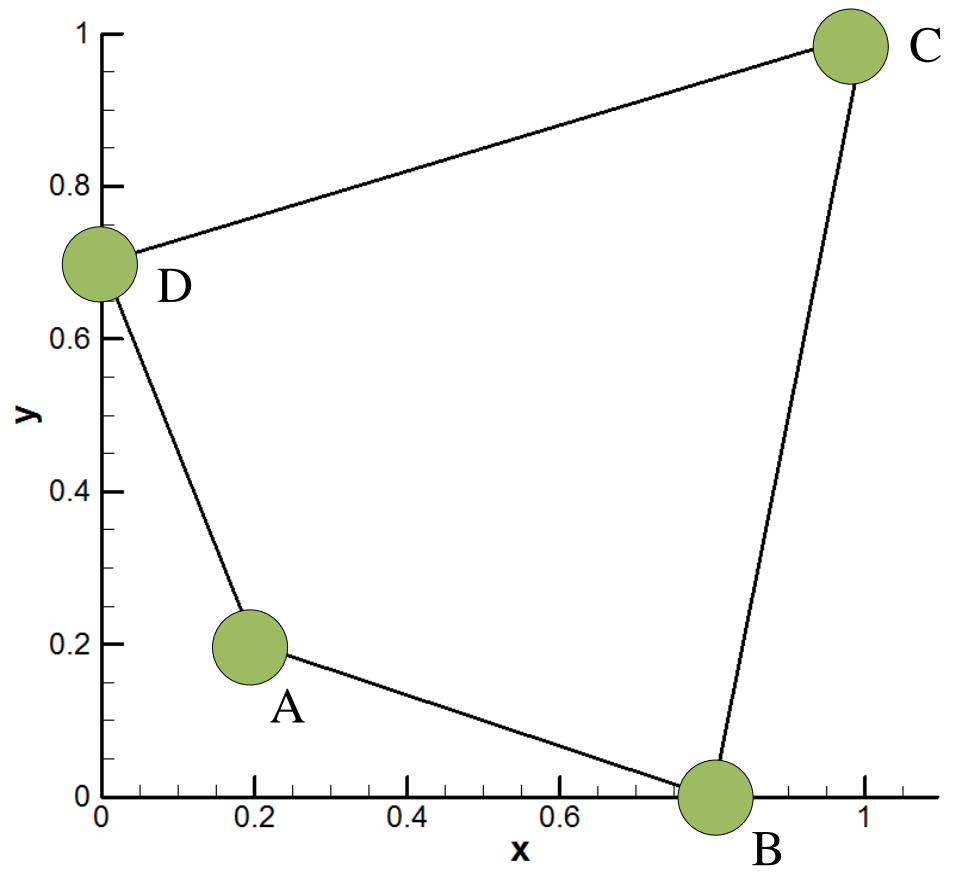

Figure 3: A general quadrilateral element 


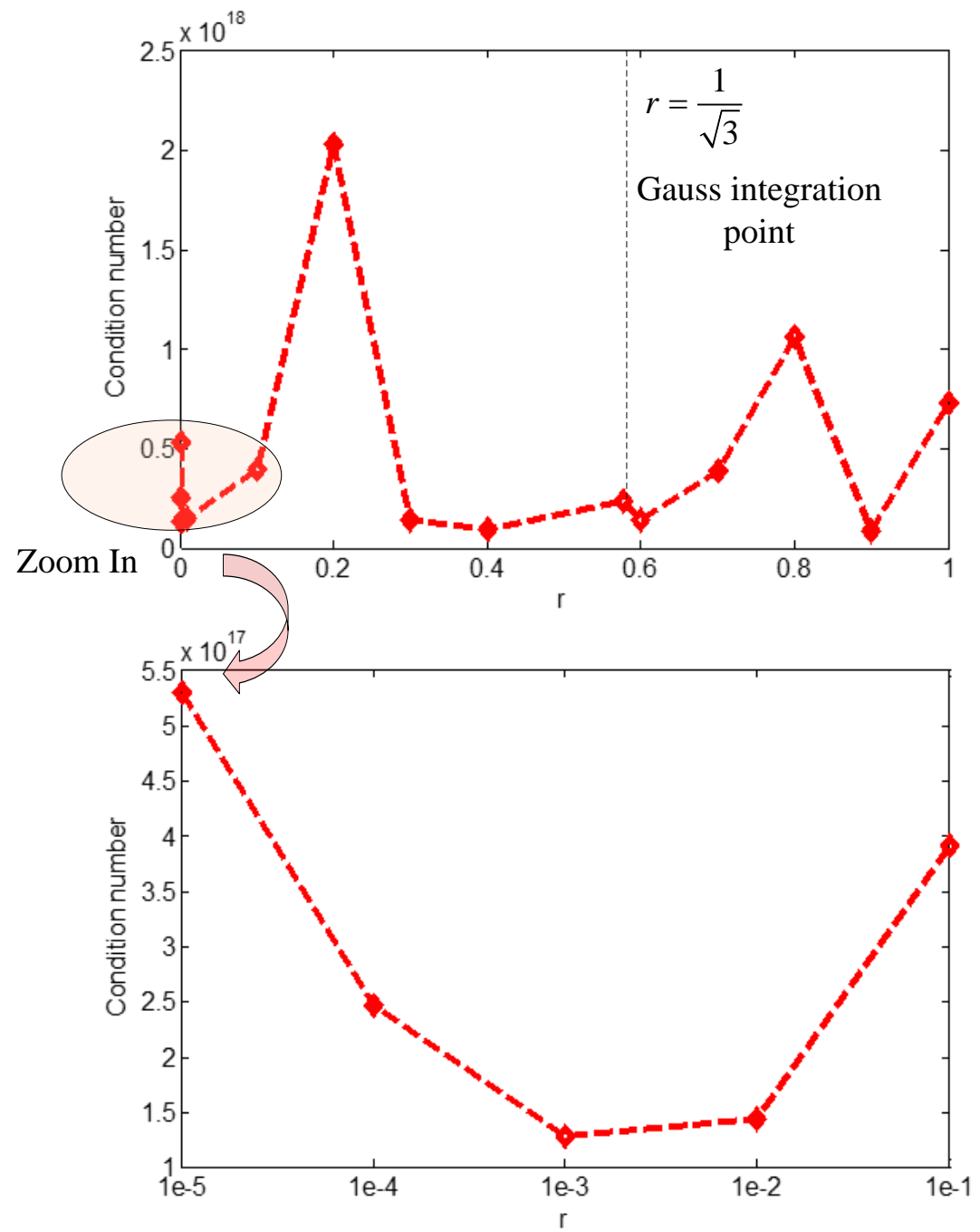

Figure 4: Condition number with various integration points (except $r=0$ ) 


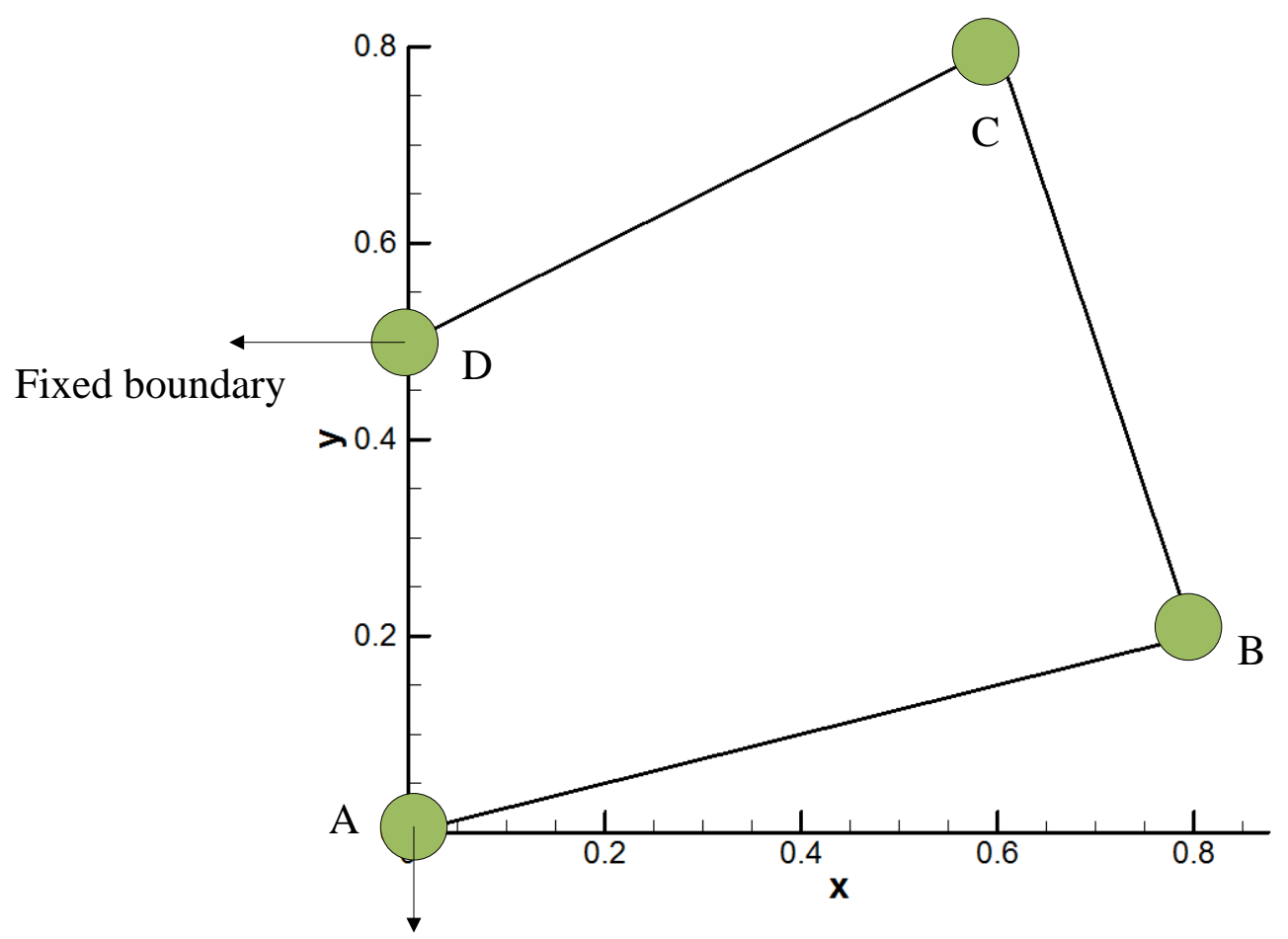

Fixed boundary

Figure 5: A general quadrilateral element with fixed boundary at two points 


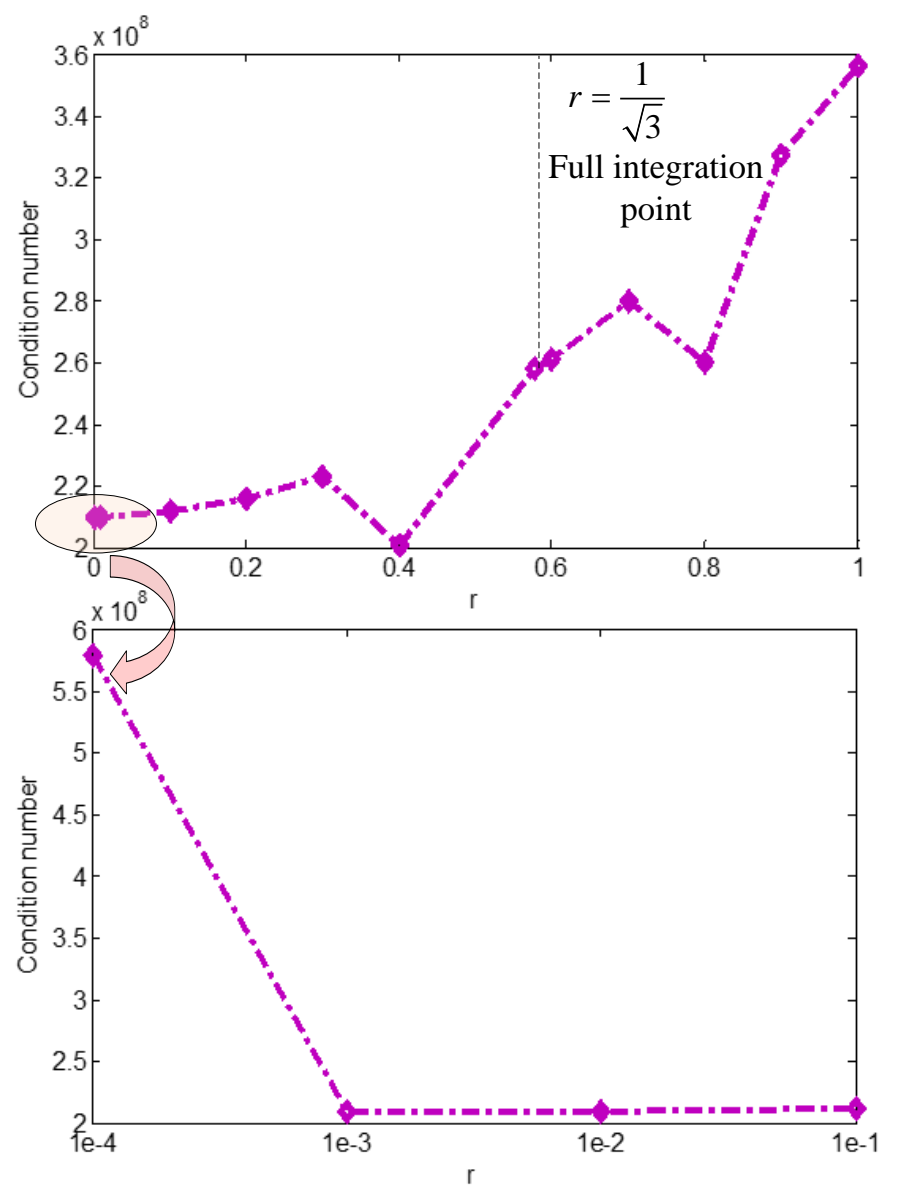

Figure 6: Condition number with various integration points (except $r=0$ ) 


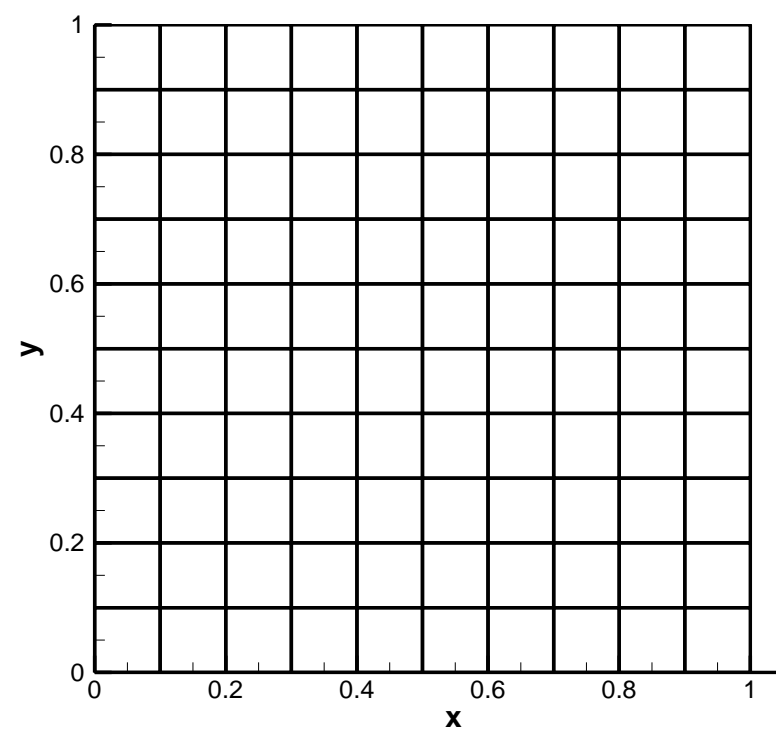

(a)

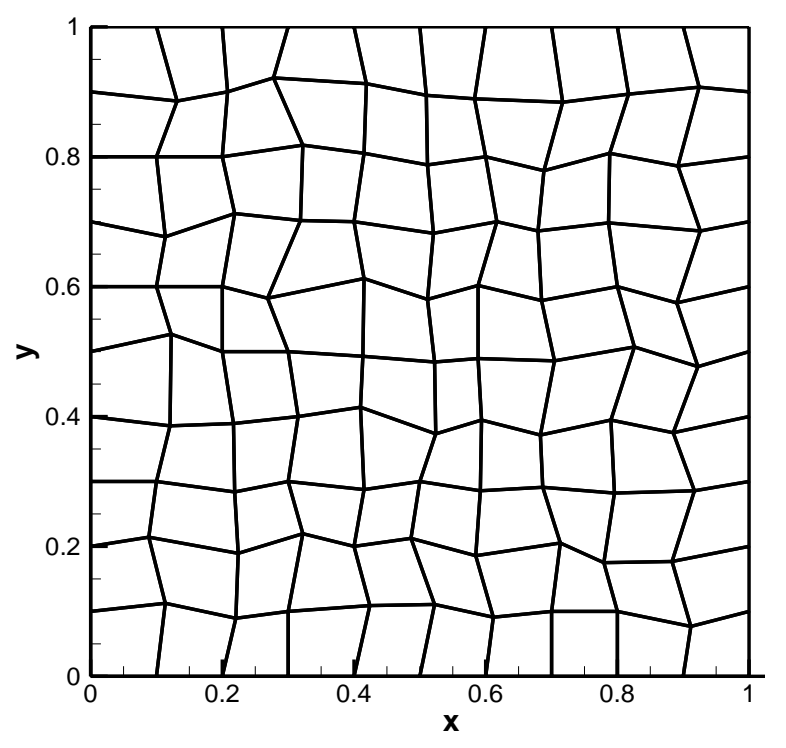

(b)

Figure 7: Mesh information 


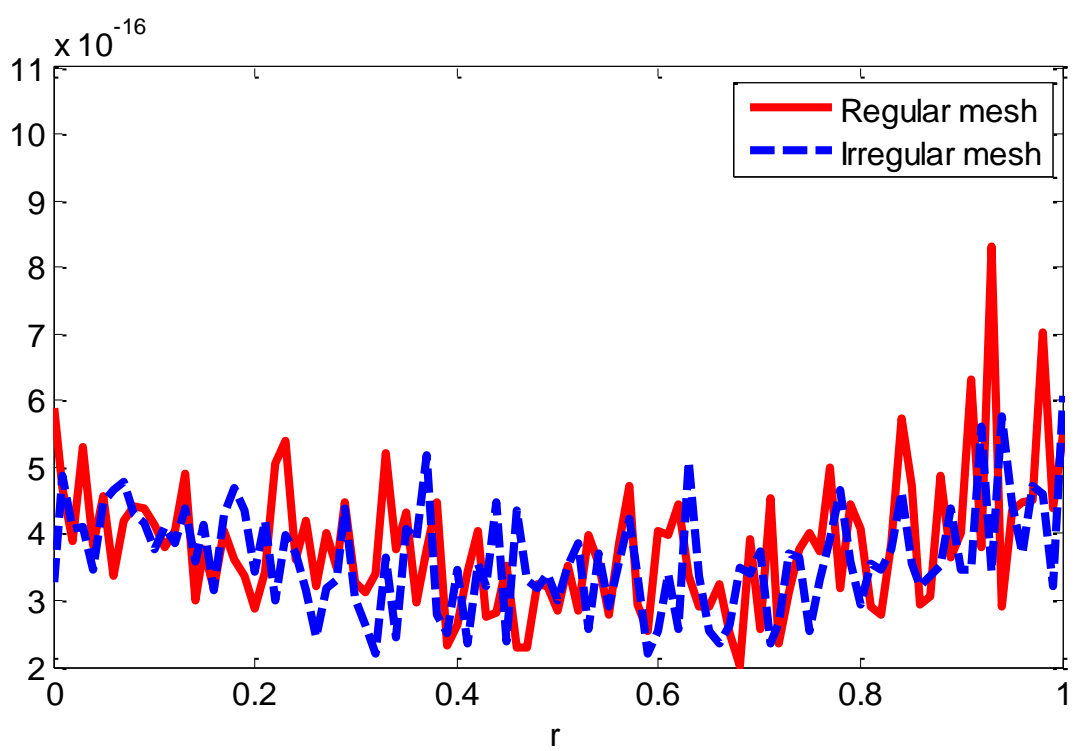

Figure 8: Displacement error for various integration points $r$ 


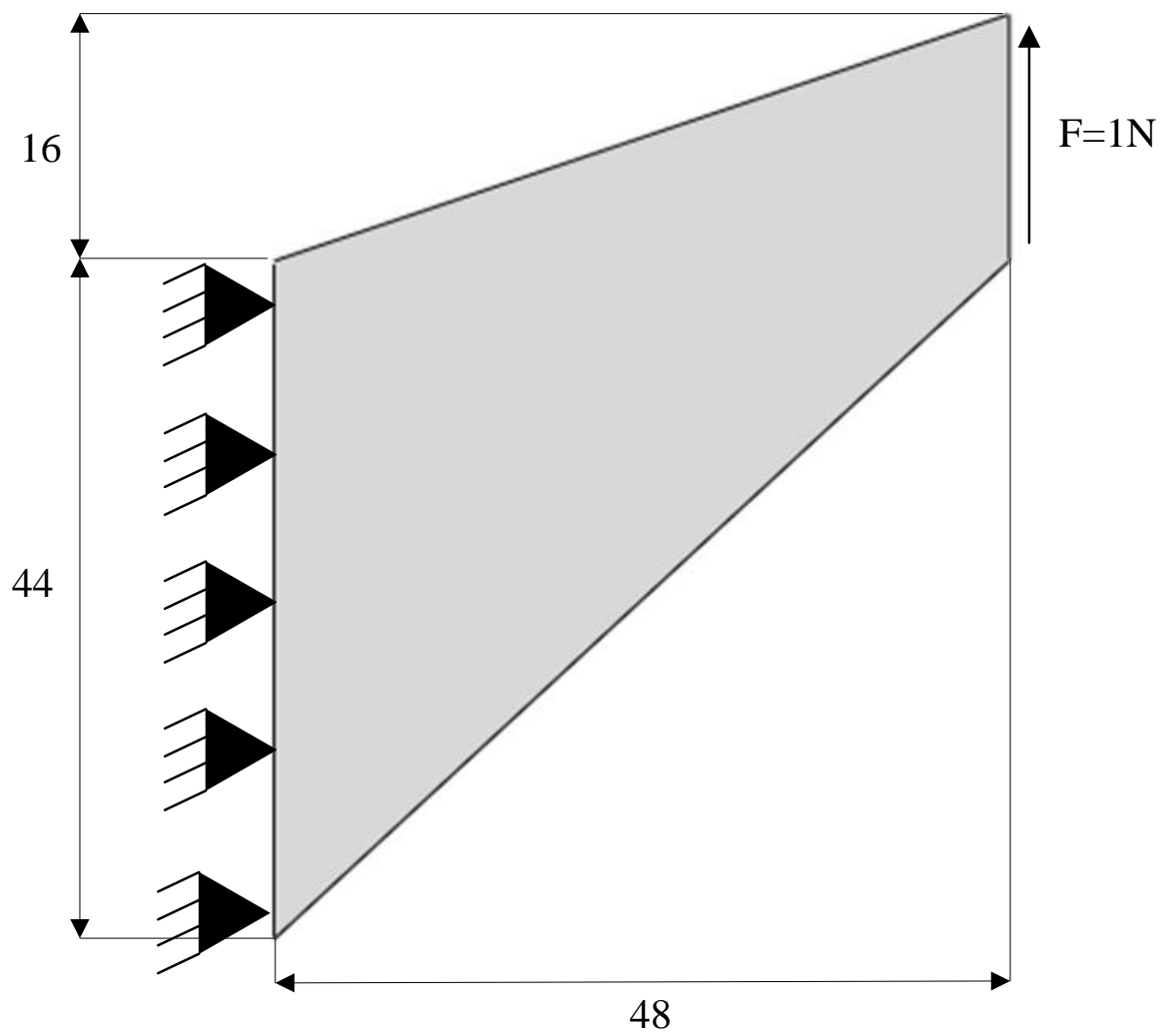

Figure 9: Cook membrane 


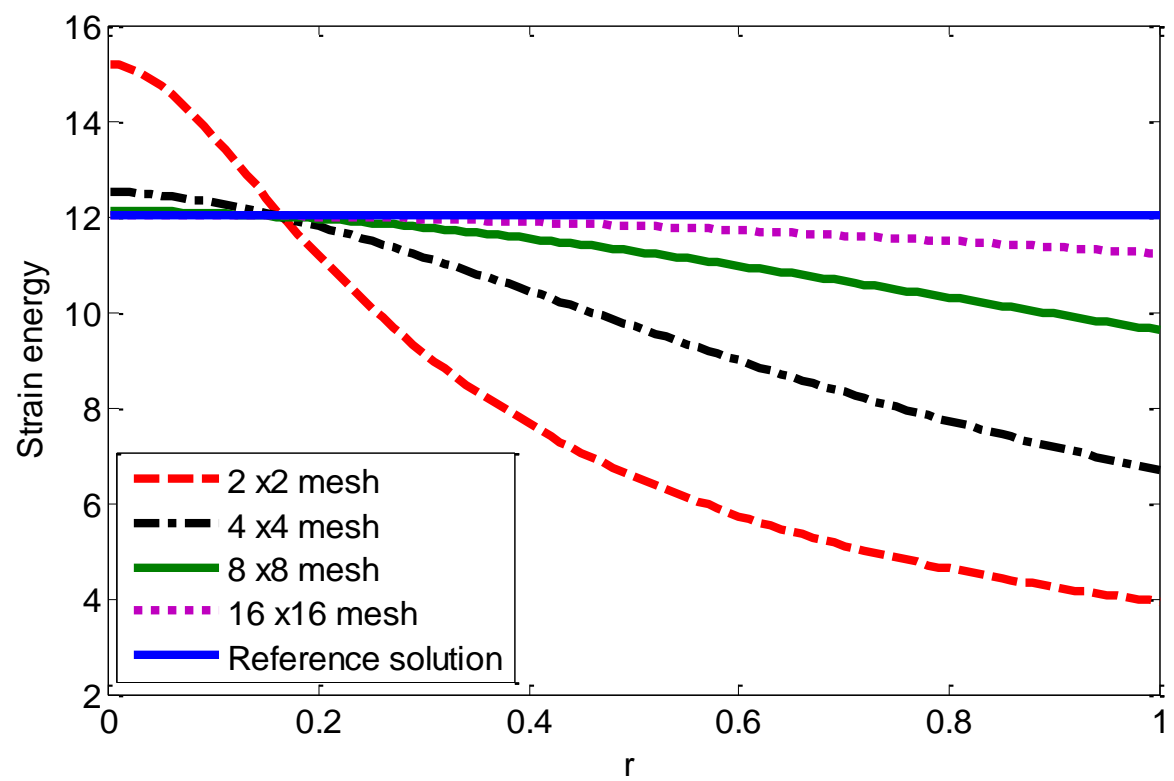

Figure 10: Strain energy for Cook's membrane problem 


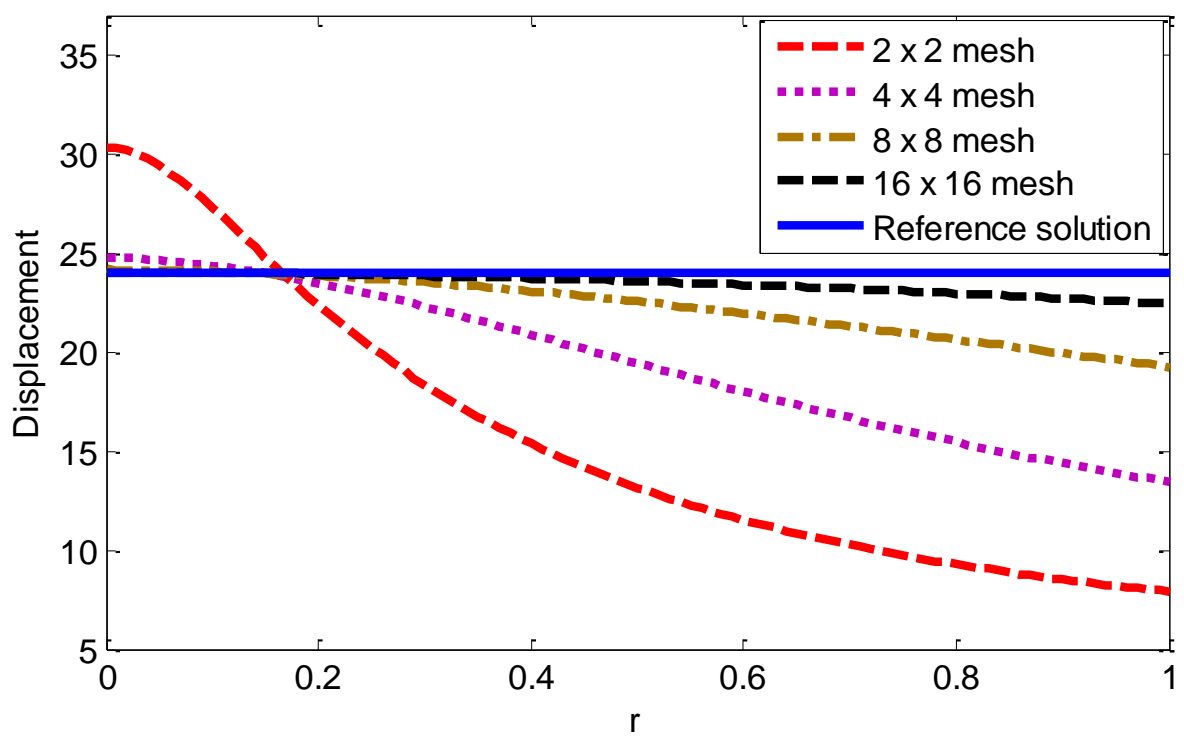

Figure 11: Displacement for Cook's membrane problem 


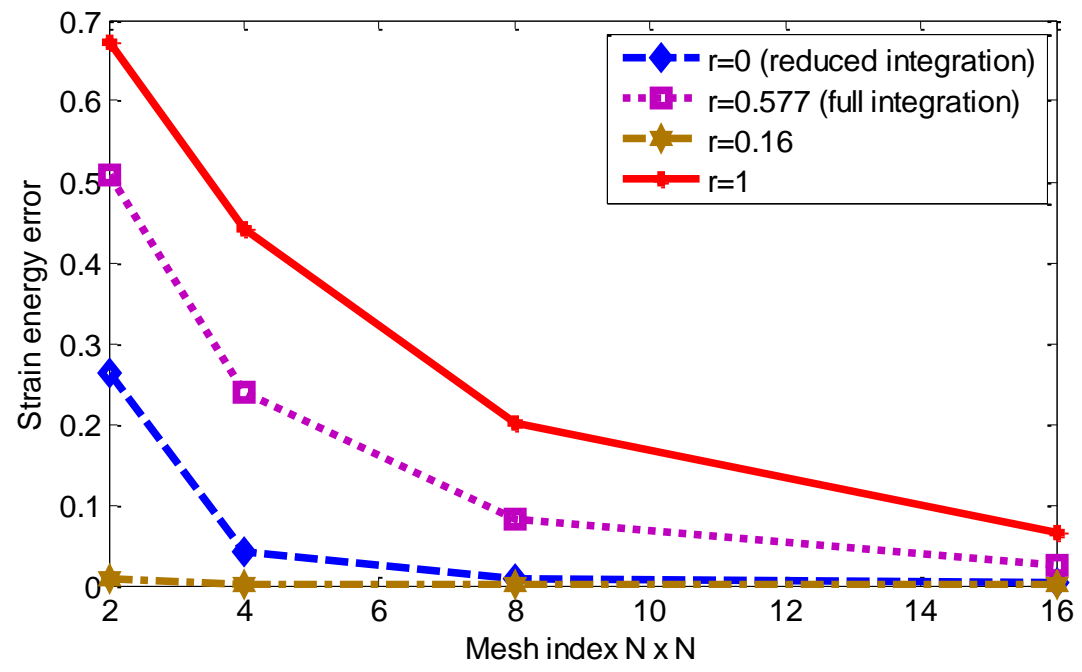

Figure 12: Convergence of strain energy for Cook's membrane problem 


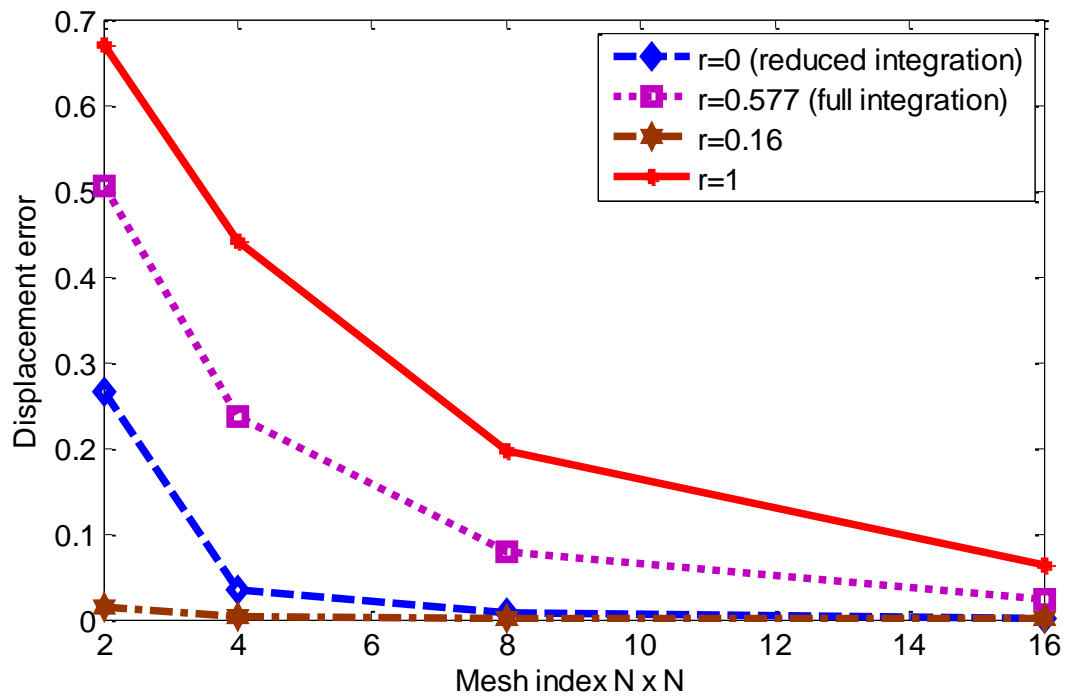

Figure 13: Convergence of displacement for Cook's membrane problem 


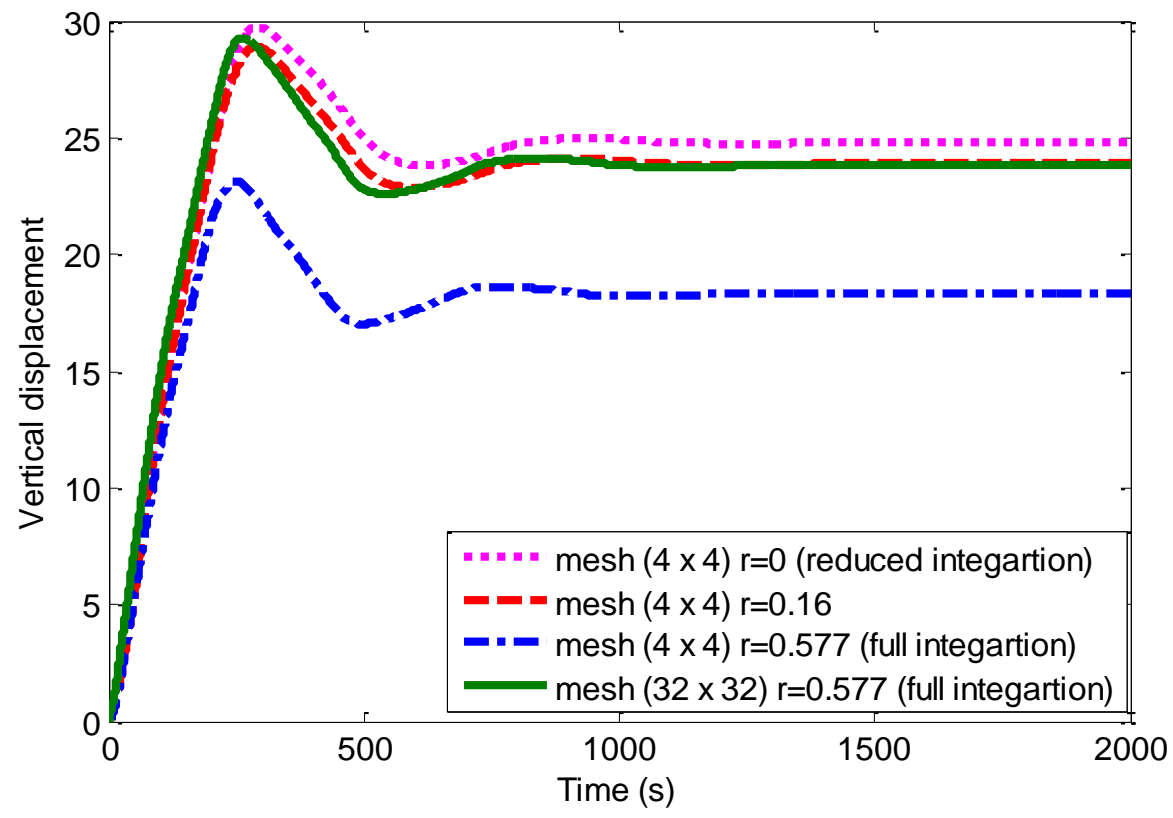

Figure 14: Vertical displacement with time 


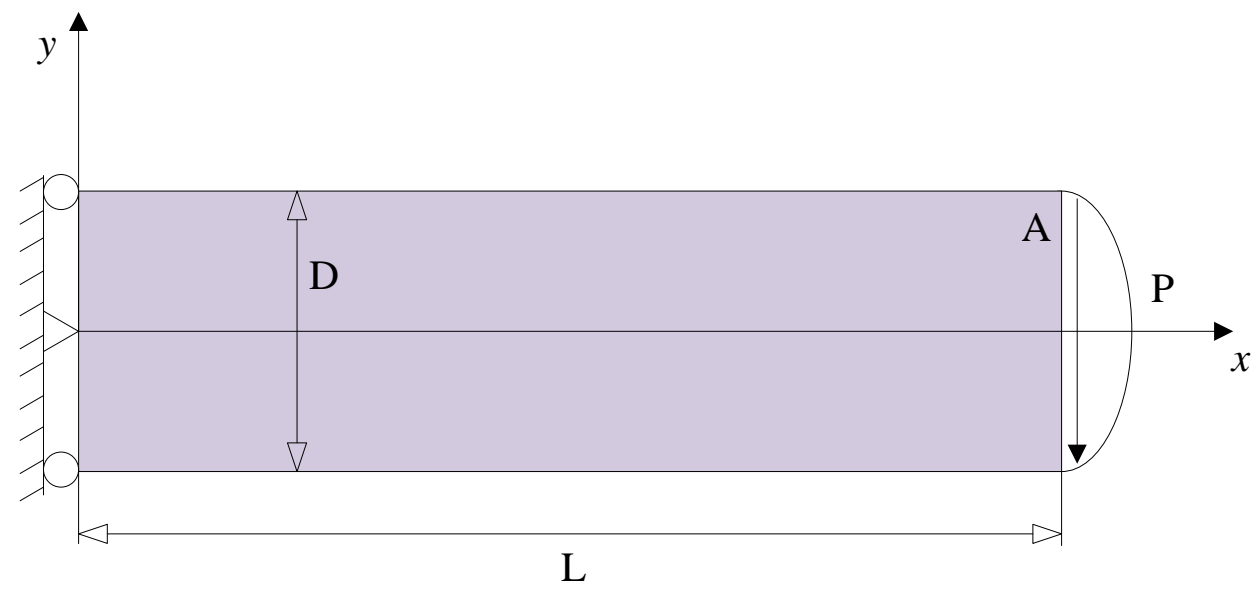

Figure 15: Model of the cantilever subjected to traction 


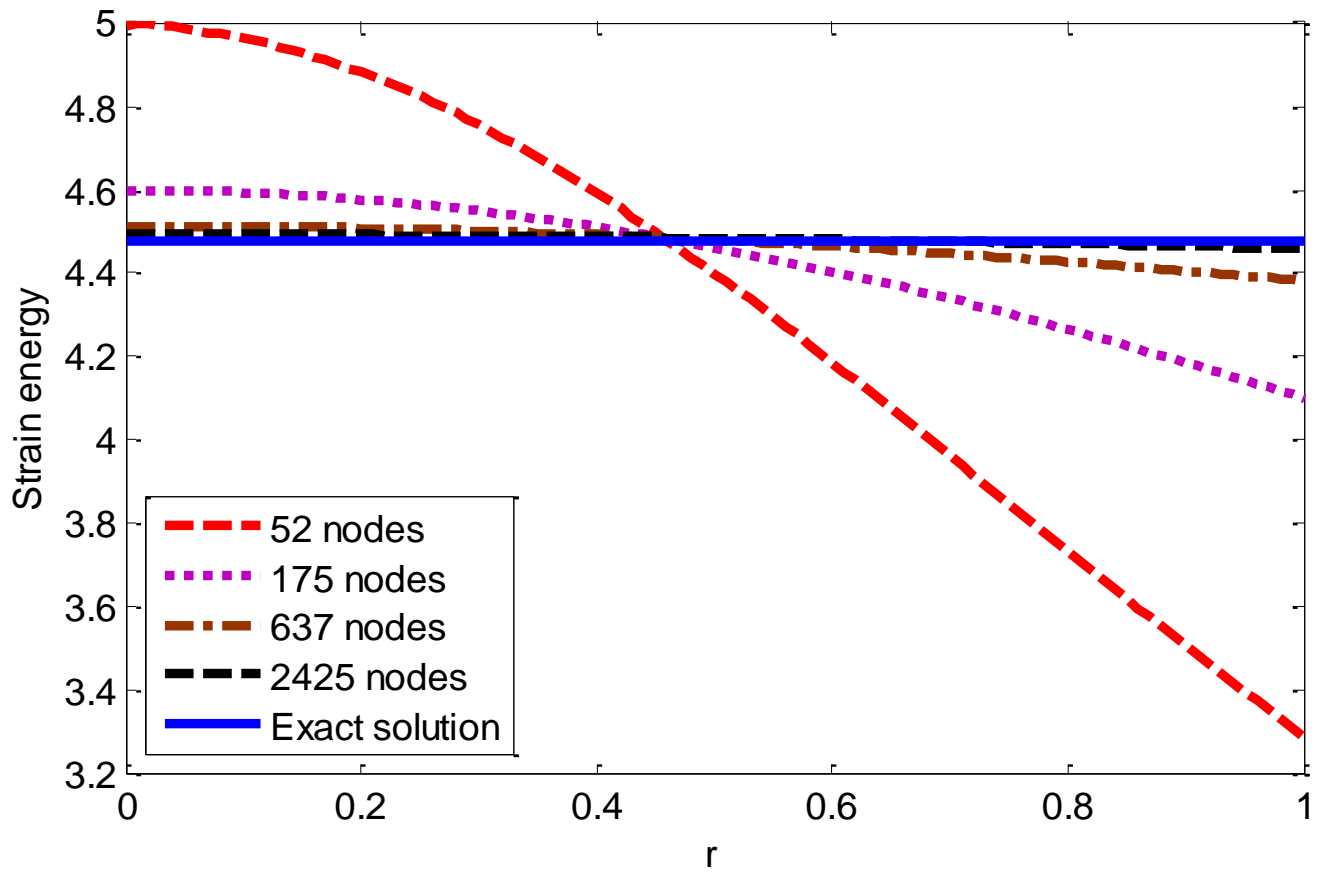

Figure 16: Strain energy with various integration points 


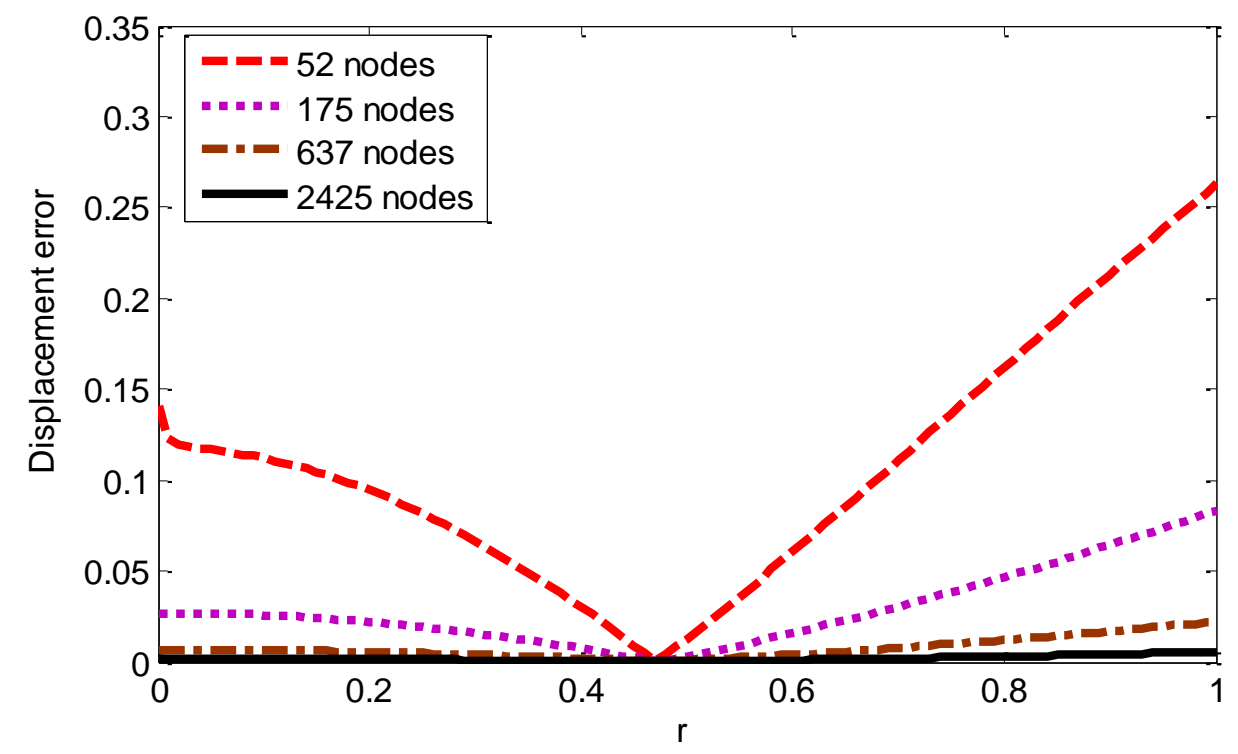

Figure 17: Displacement error with various integration points 


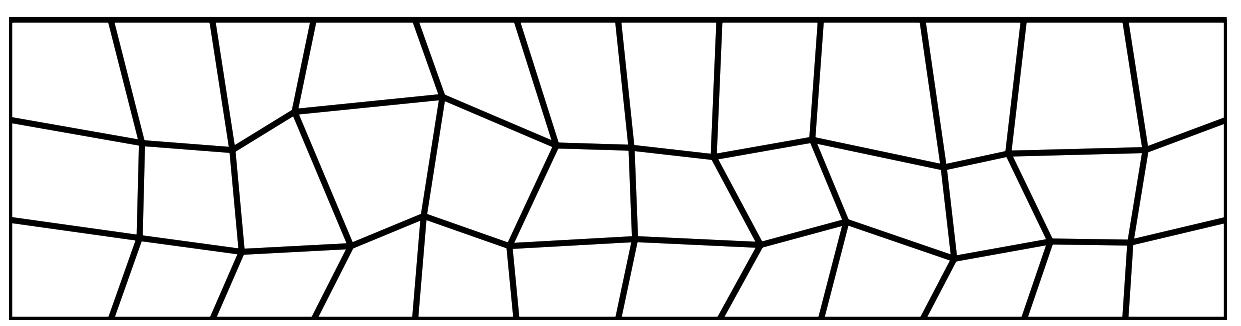

(a) 52 nodes

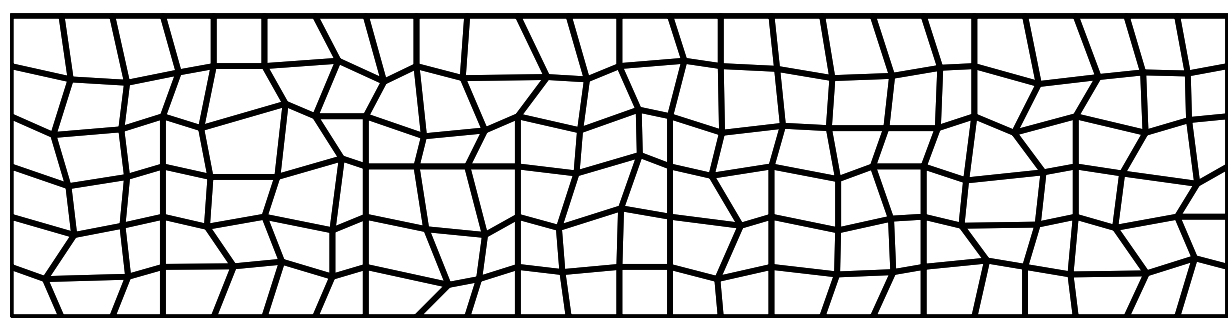

(b) 175 nodes

Figure 18: Irregular mesh 


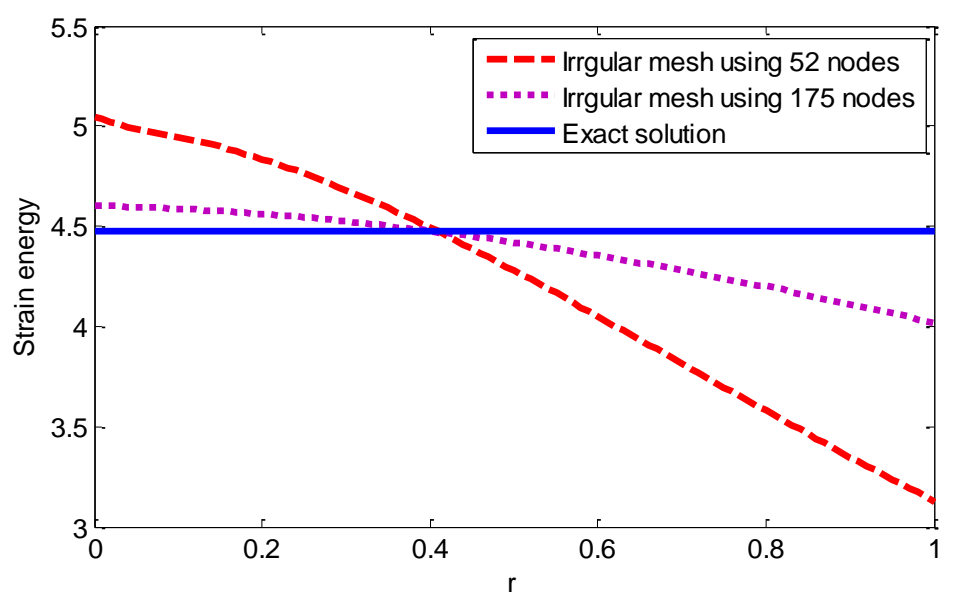

(a) Strain energy

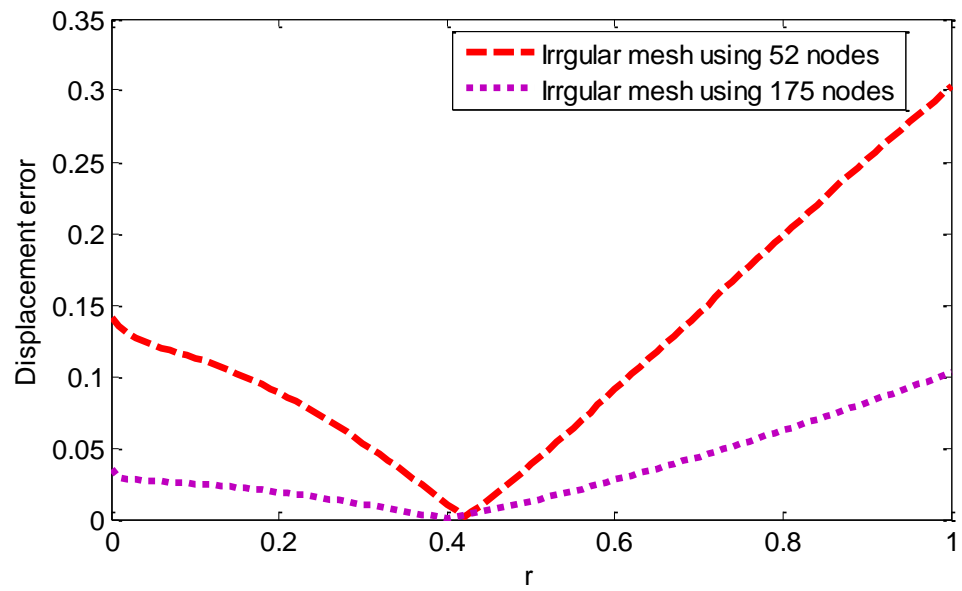

(b) Displacement error

Figure 19: Numerical results using irregular mesh 


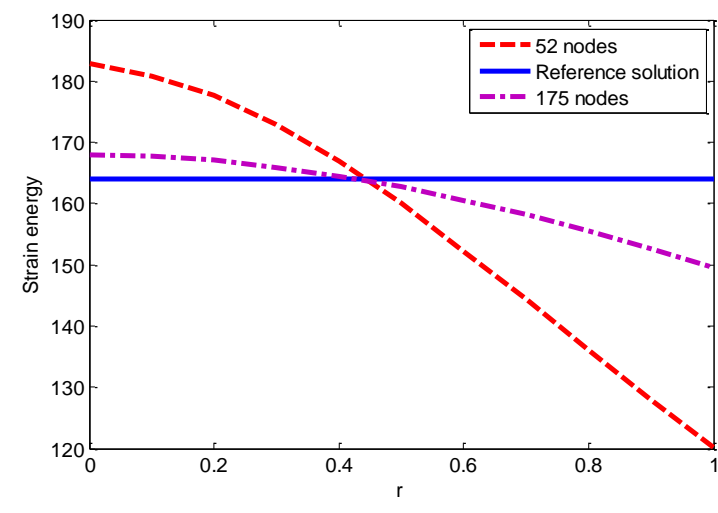

(a) Strain energy

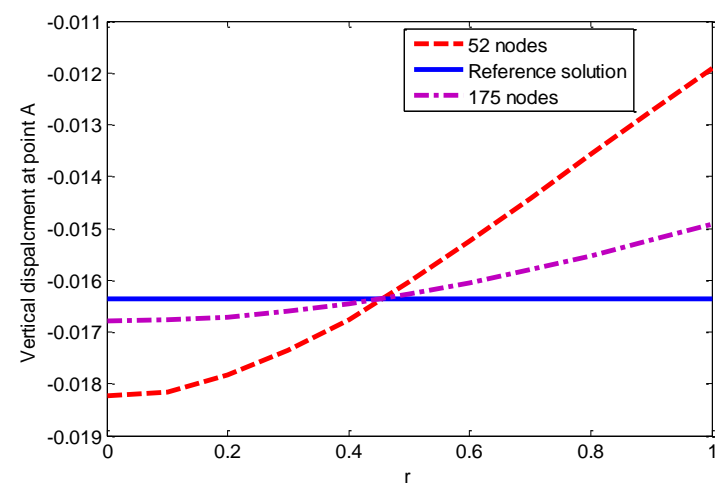

(b) Vertical displacement at point $\mathrm{A}$

Figure 20: Optimal integration point $r$ in the static case 


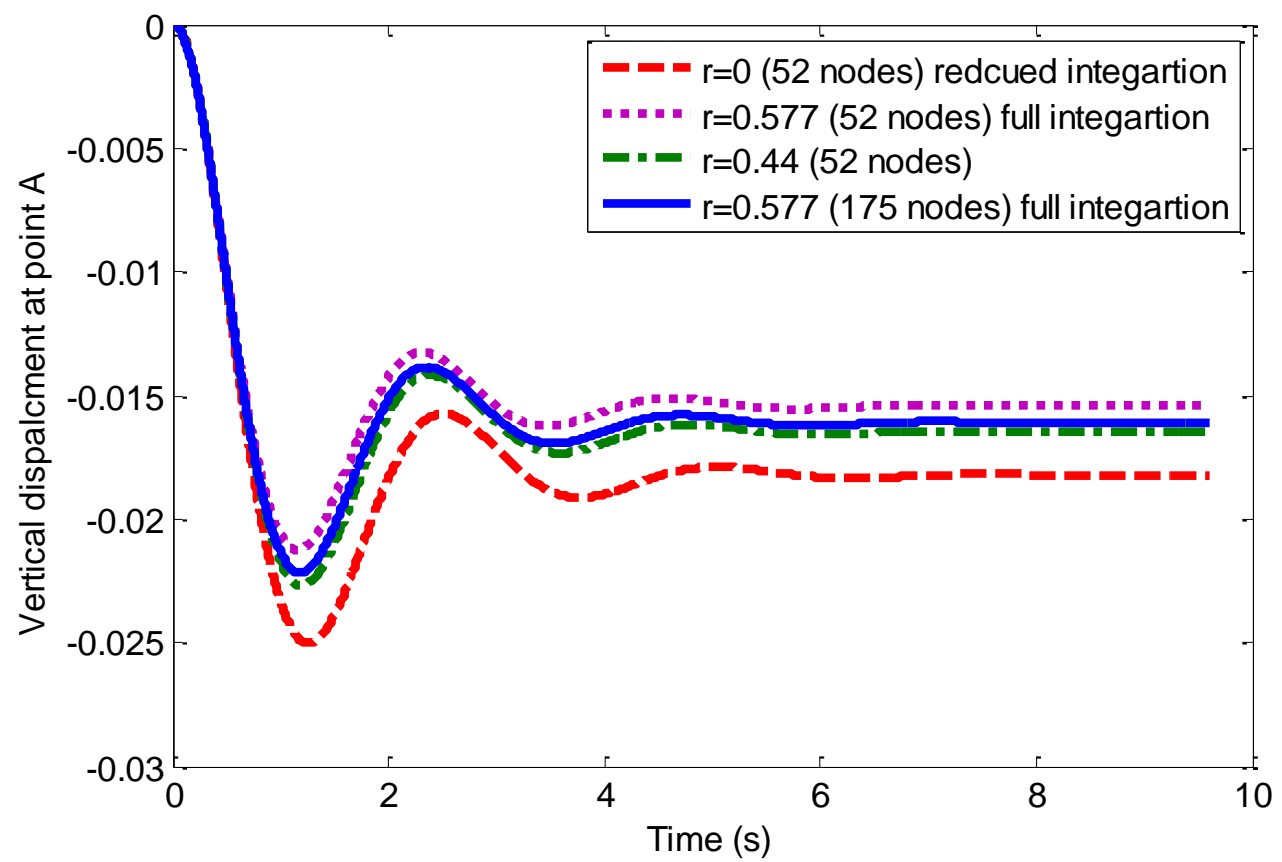

Figure 21: Vertical displacement with time 


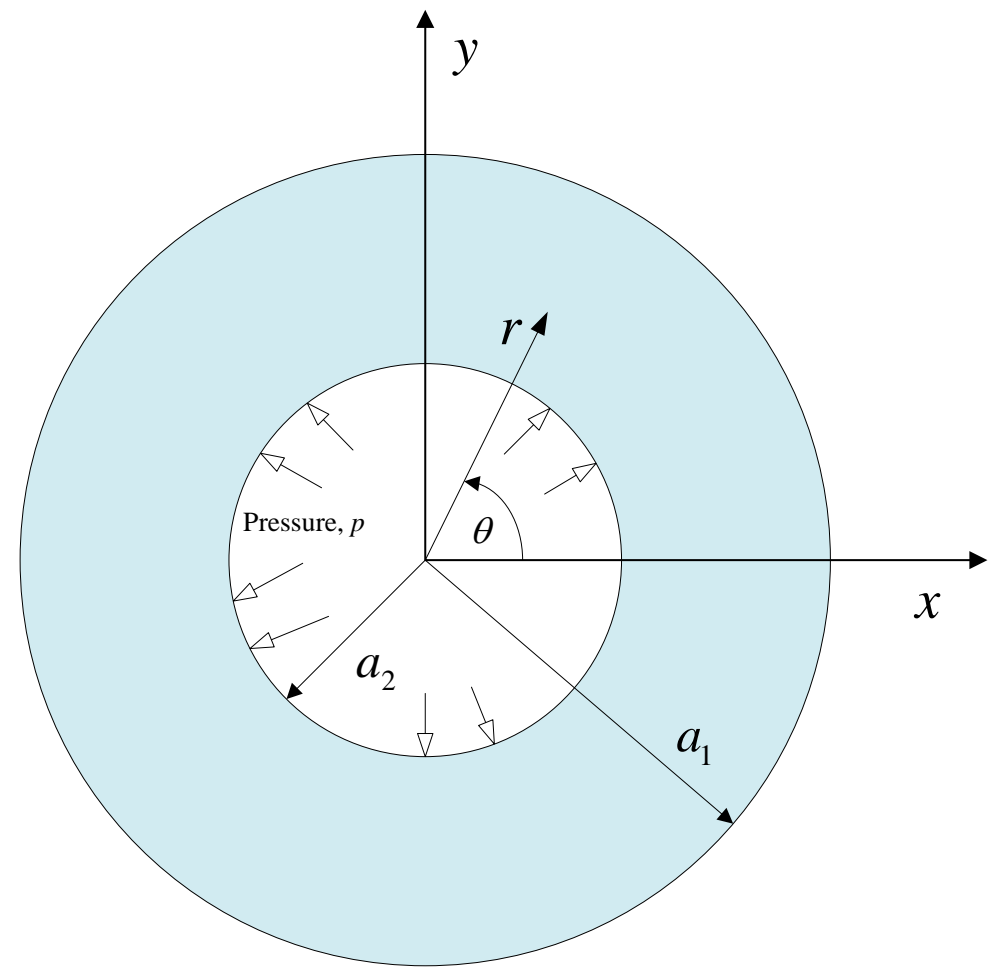

(a) Full model

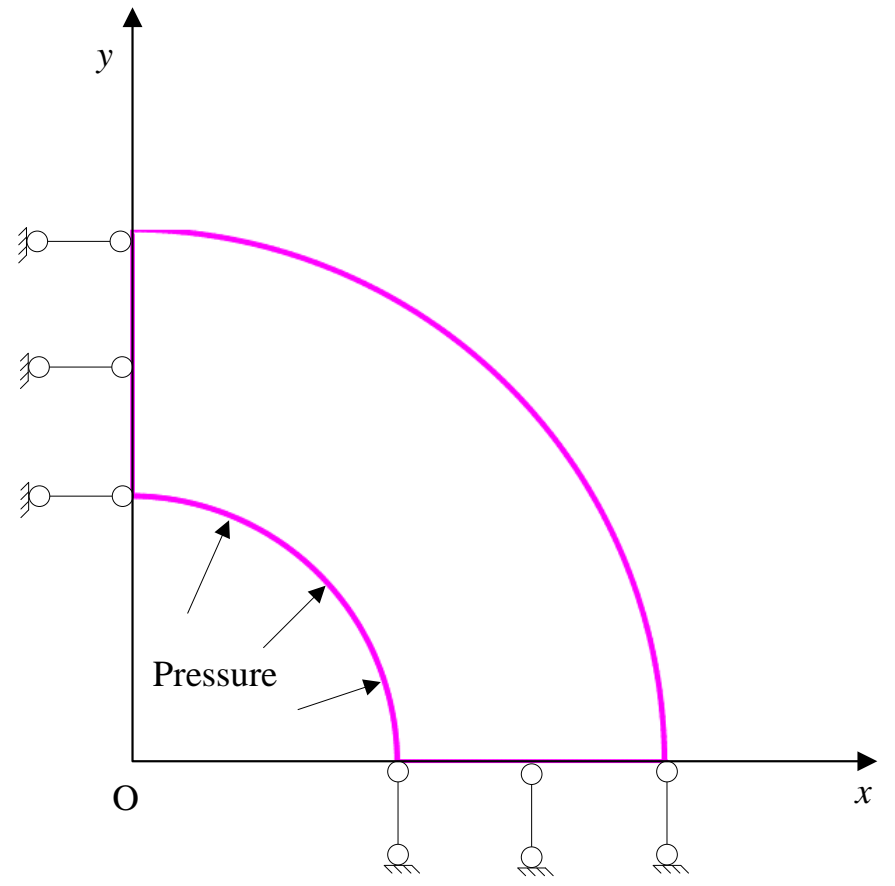

(b) One quarter model

Figure 22: Cylindrical pipe subjected to an inner pressure 


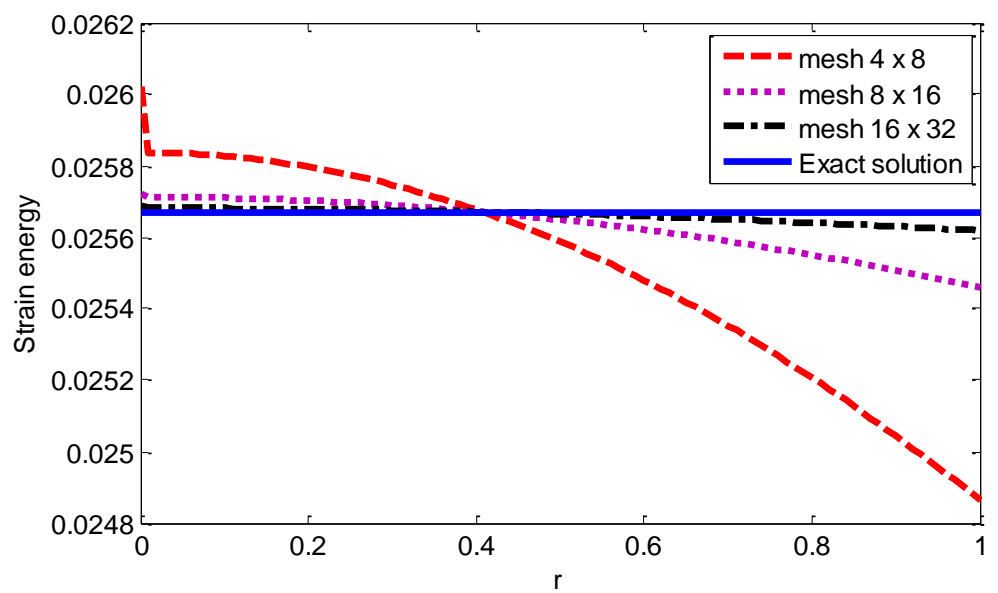

Figure 23: Strain energy with various integration points 


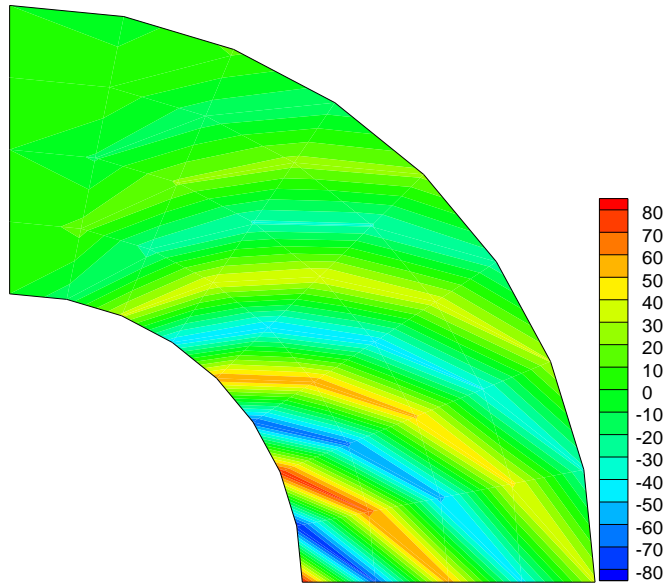

(a) $r=0$ (reduced integration)

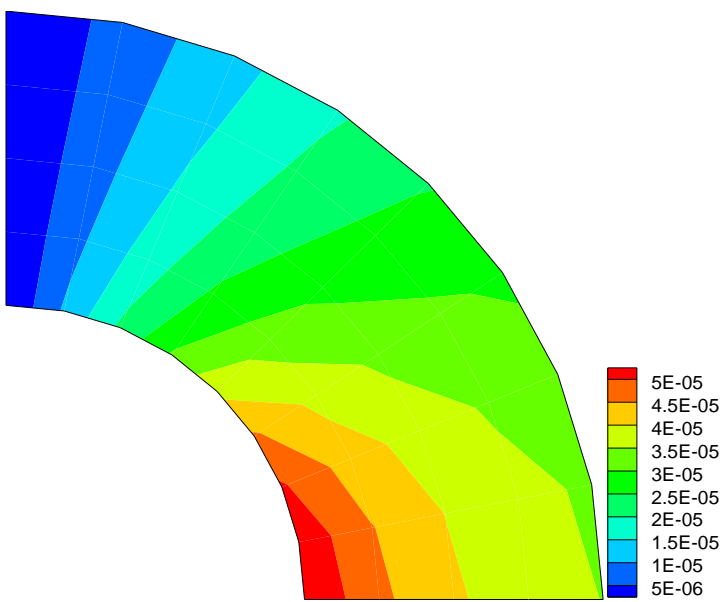

(c) $\mathrm{r}=0.001$

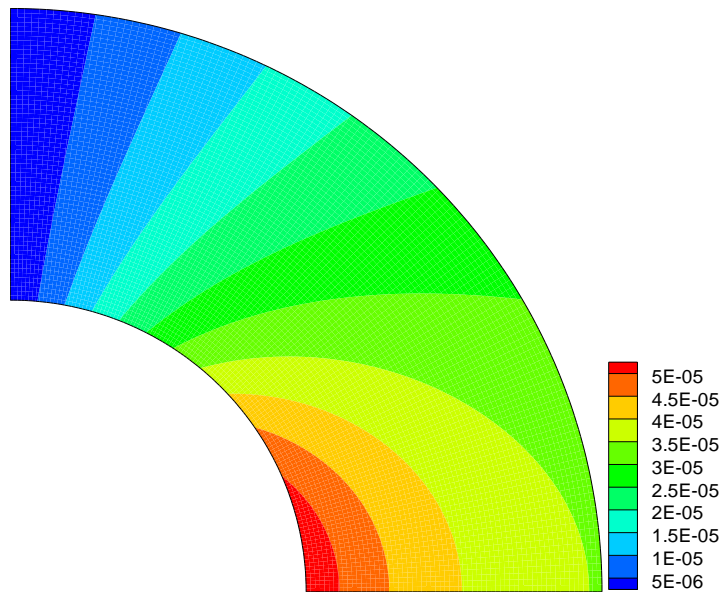

(b) Exact solution

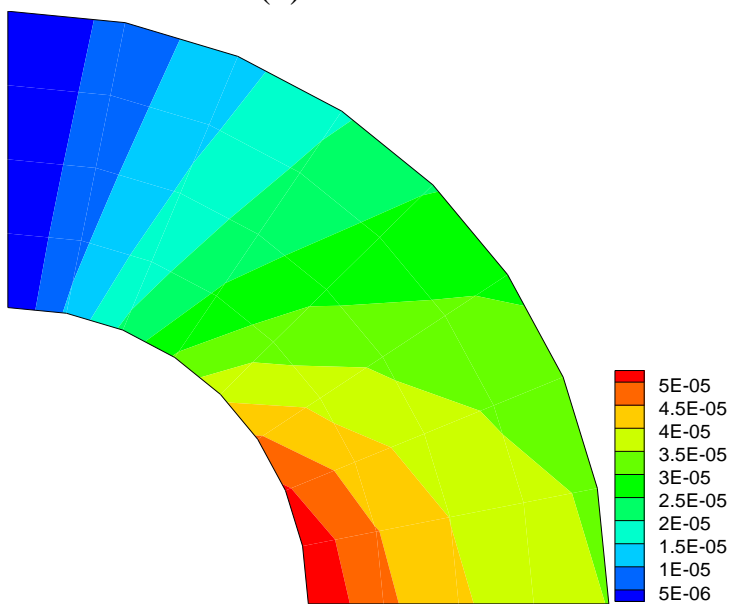

(d) $\mathrm{r}=0.01$

Figure 24: Comparison of displacement contour along $x$ direction 


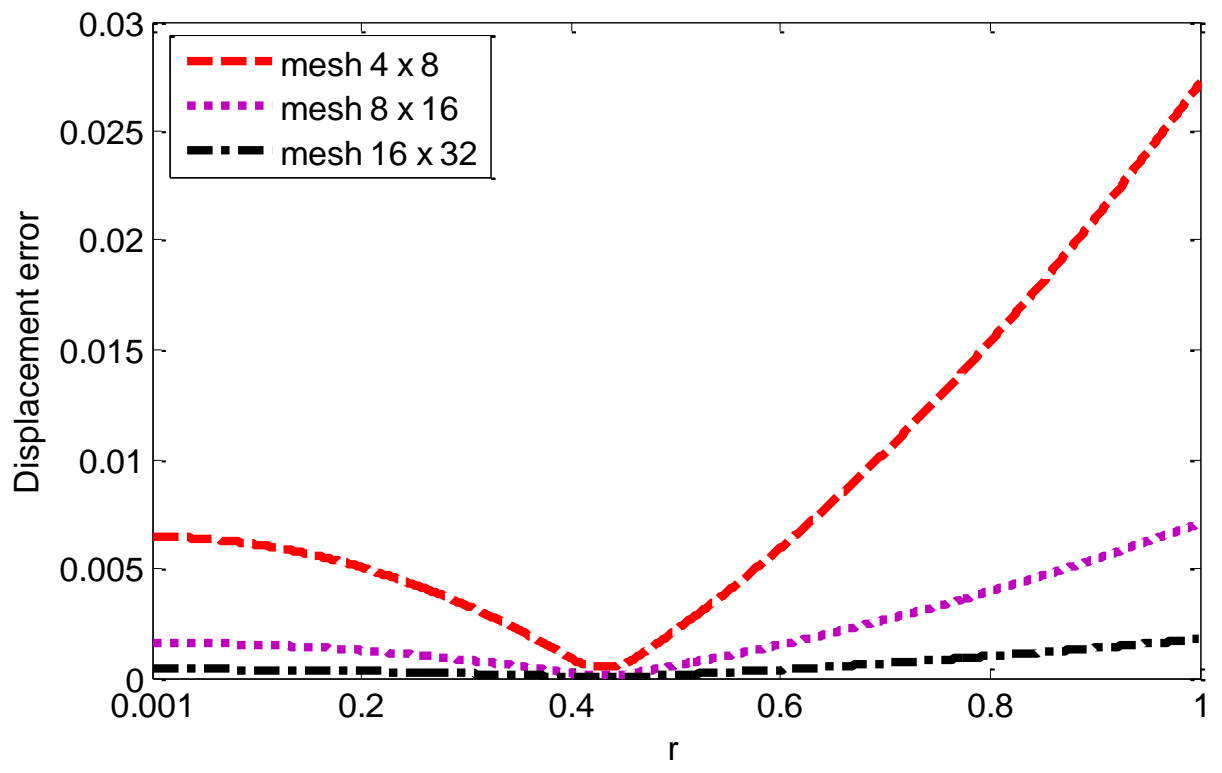

Figure 25: Displacement error with various integration points 


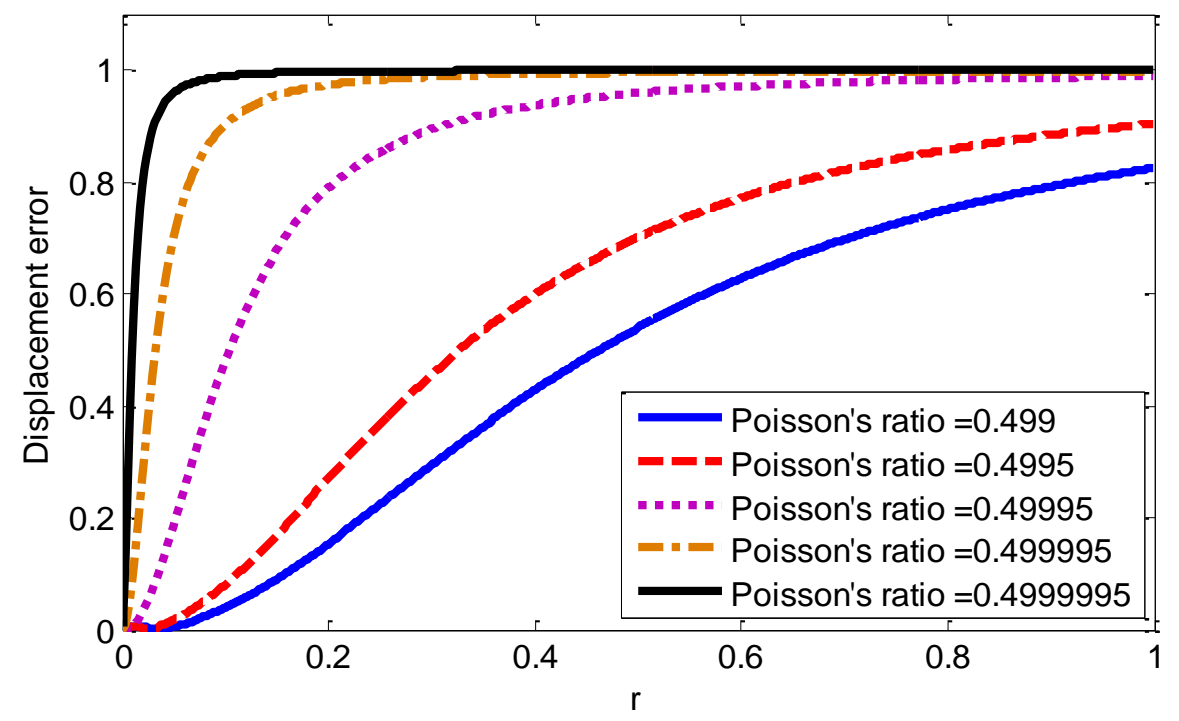

Figure 26: Displacement error for various integration points (Poison's ratio $v=0.499$ ) 


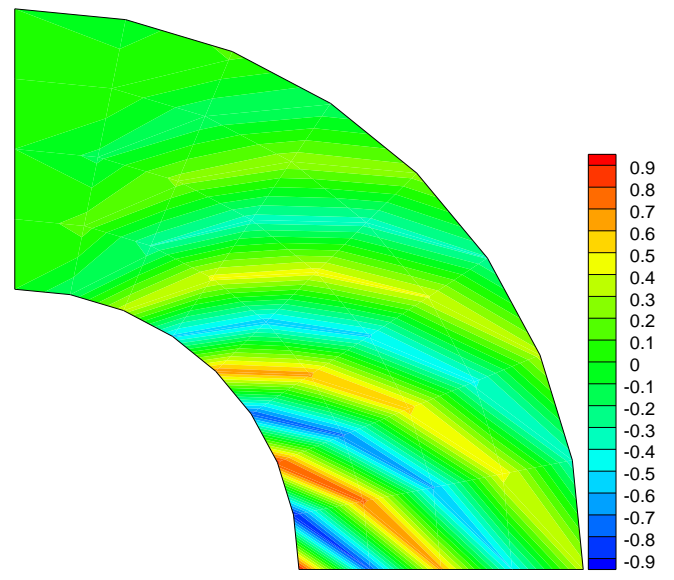

(a) Poisson's ratio v $=0.4995$

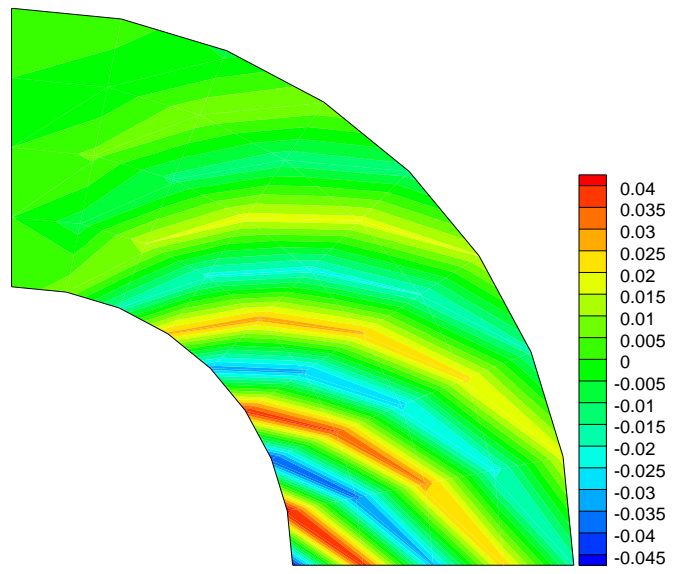

(c) Poisson's ratio $v=0.499995$

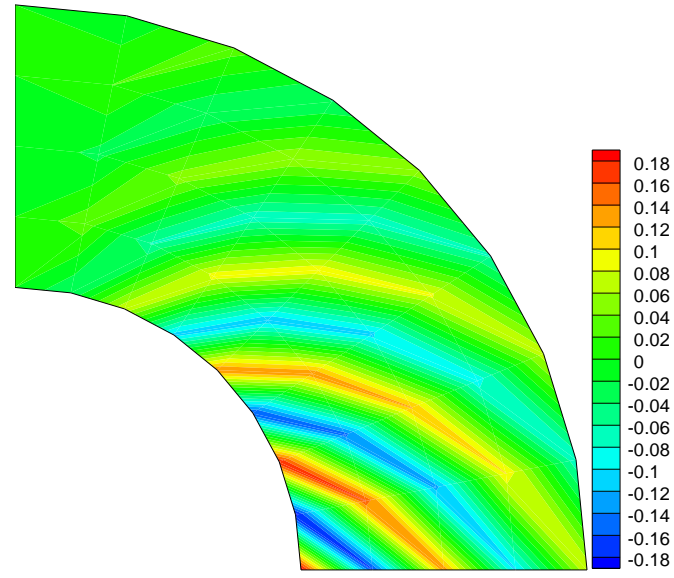

(b) Poisson's ratio v $=0.49995$

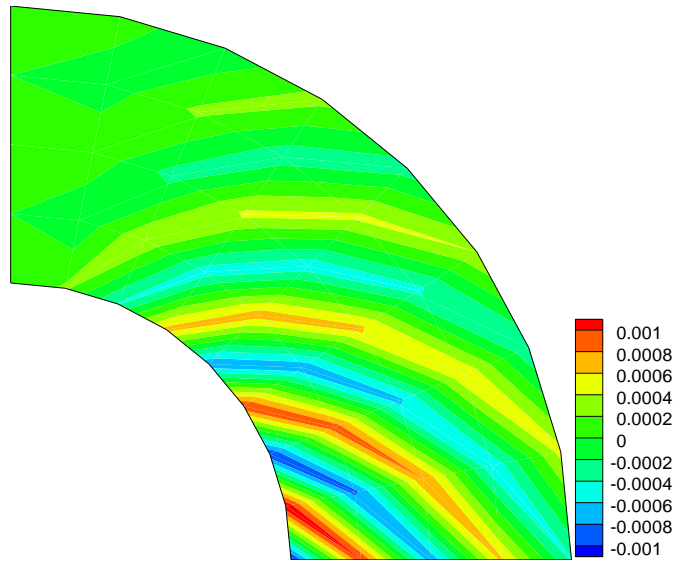

(d) Poisson's ratio v $=0.499995$

Figure 27: Displacement contour along $x$ direction using reduced integration 


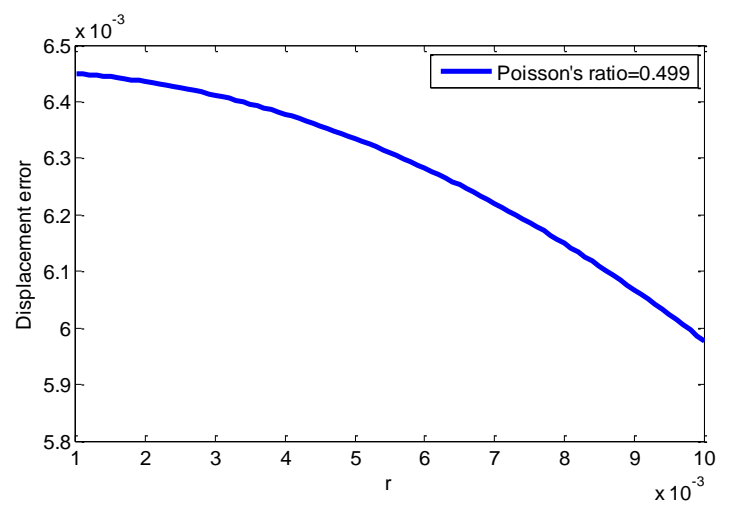

(a) $v=0.499$

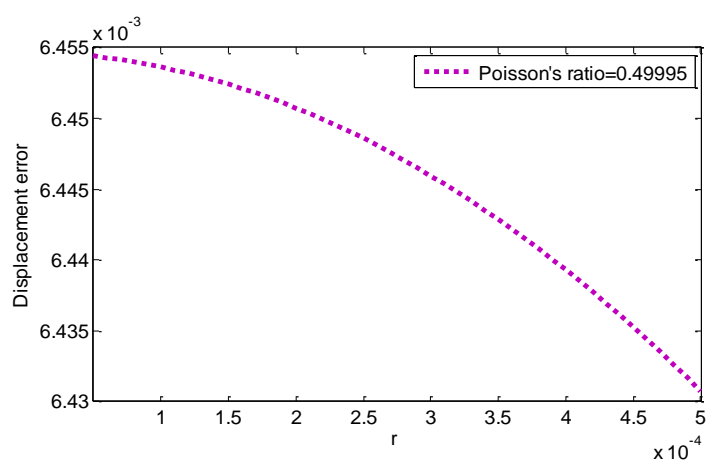

(c) $v=0.49995$

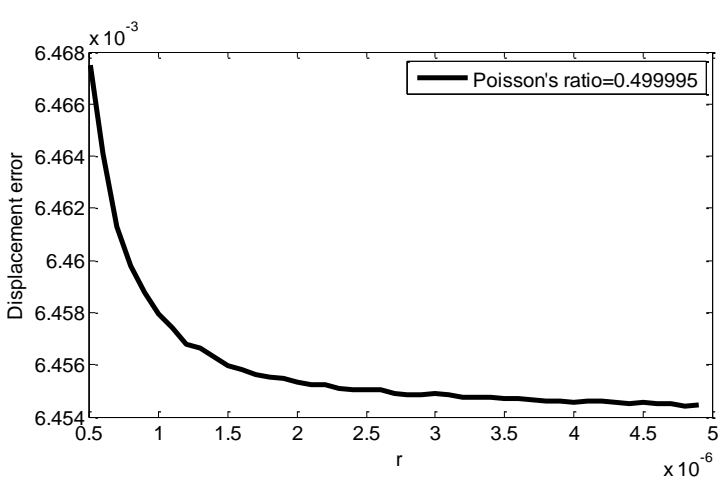

(e) $v=0.4999995$

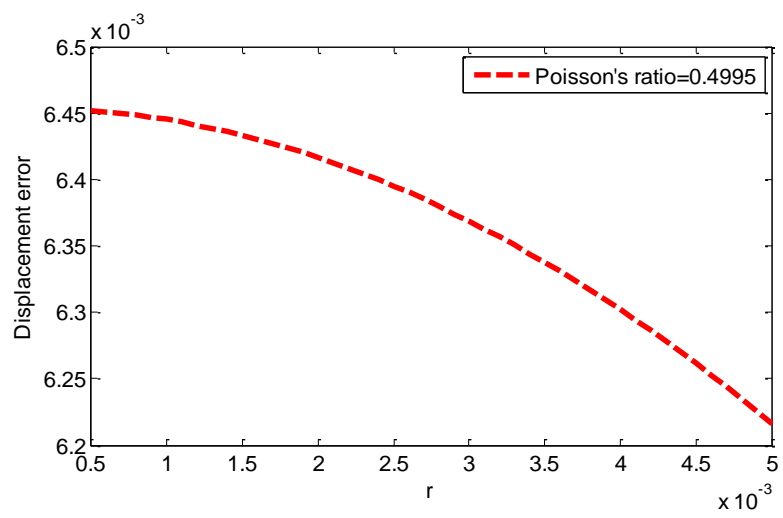

(b) $v=0.4995$

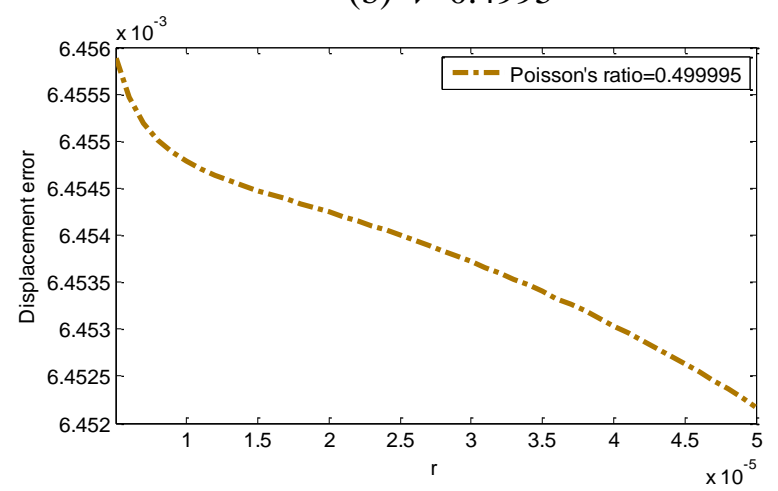

(d) $v=0.499995$

Stable range $\in[0.5-v,(0.5-v) \times 10]$

Case a $\in\left[10^{-3}, 10^{-2}\right]$

Case $b \in\left[5 \times 10^{-4}, 5 \times 10^{-3}\right]$

Case c $\in\left[5 \times 10^{-5}, 5 \times 10^{-4}\right]$

Case $d \in\left[5 \times 10^{-6}, 5 \times 10^{-5}\right]$

Case e $\in\left[5 \times 10^{-7}, 5 \times 10^{-6}\right]$

Figure 28: Displacement errors under the stable range 


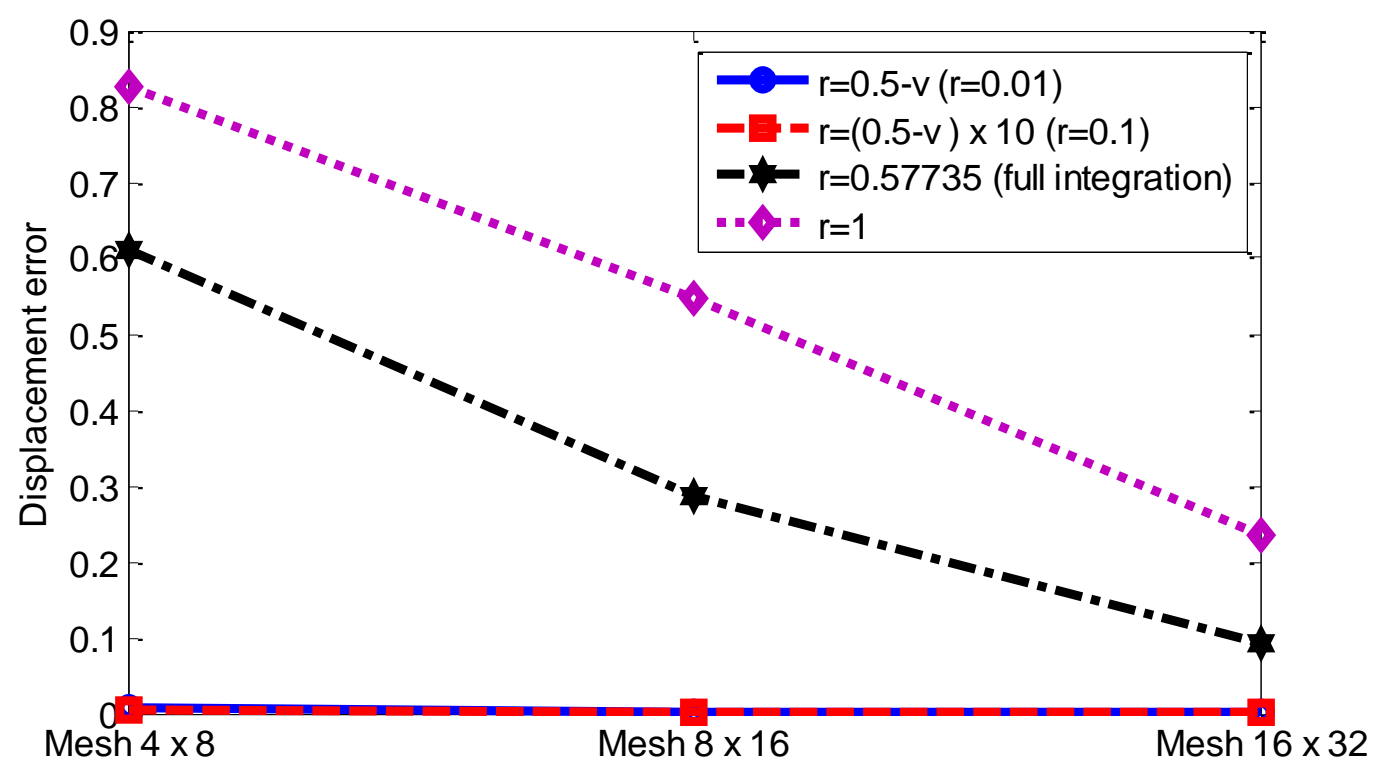

(a) Poisson's ratio $v=0.499$

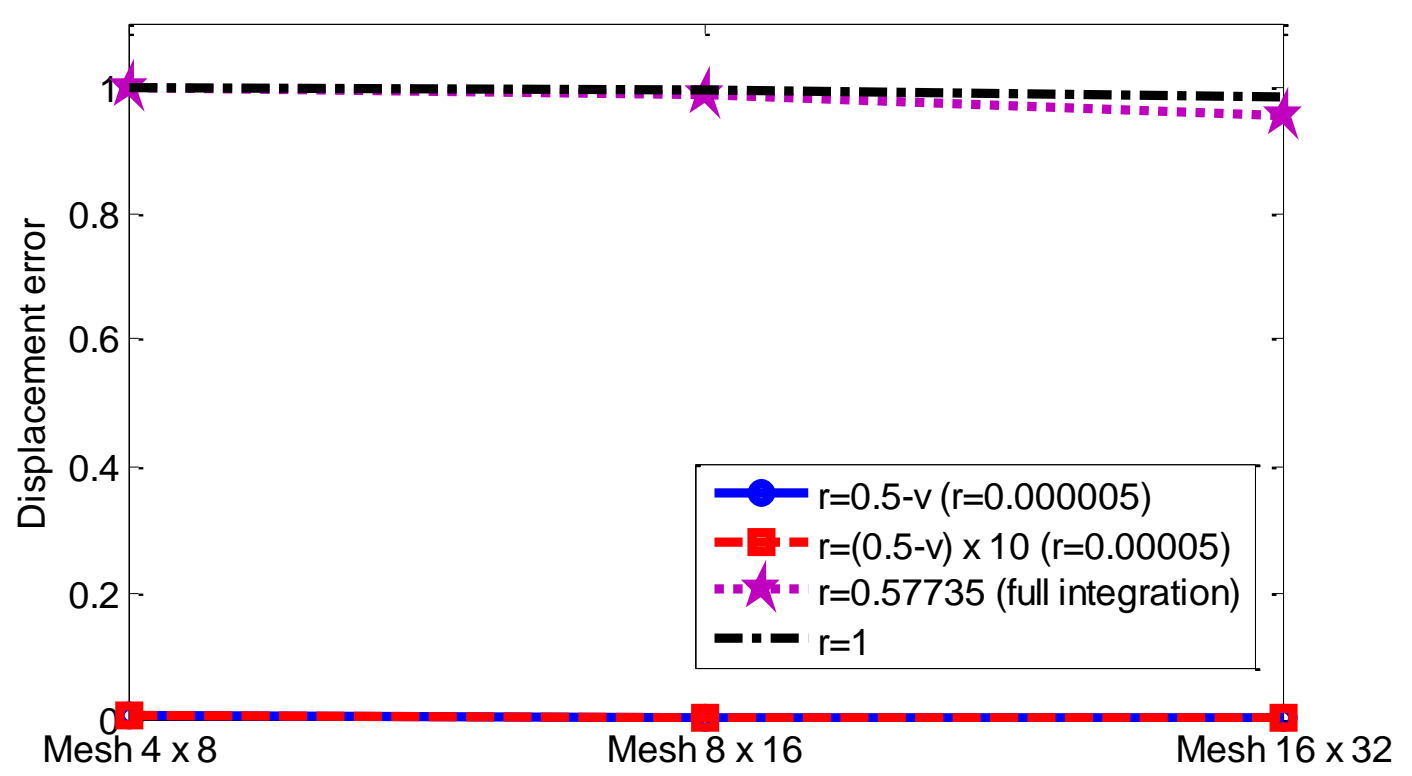

(b) Poisson's ratio $v=0.499995$

Figure 29: Convergence rate of different integration points 


\section{Table}

Table 1: Effect of integartion points on the prediction of Eigenvalue (compressible case)

\begin{tabular}{ccccccccc}
\hline \hline $\begin{array}{c}\text { Integration } \\
\text { points }\end{array}$ & 1 & 2 & 3 & 4 & 5 & 6 & 7 & 8 \\
\hline$r=0$ & 0 & 0 & 0 & 0 & 0 & $2.29 \mathrm{e} 7$ & $2.32 \mathrm{e} 7$ & $5.83 \mathrm{e} 7$ \\
$r=1 \mathrm{e}-5$ & 0 & 0 & 0 & $5.00 \mathrm{e}-2$ & $5.88 \mathrm{e}-3$ & $2.29 \mathrm{e} 7$ & $2.32 \mathrm{e} 7$ & $5.83 \mathrm{e} 7$ \\
$r=1 \mathrm{e}-4$ & 0 & 0 & 0 & $5.00 \mathrm{e}-1$ & $5.88 \mathrm{e}-1$ & $2.29 \mathrm{e} 7$ & $2.32 \mathrm{e} 7$ & $5.83 \mathrm{e} 7$ \\
$r=1 \mathrm{e}-3$ & 0 & 0 & 0 & $5.0 \mathrm{e} 2$ & $5.88 \mathrm{e} 2$ & $2.29 \mathrm{e} 7$ & $2.32 \mathrm{e} 7$ & $5.83 \mathrm{e} 7$ \\
$r=1 \mathrm{e}-2$ & 0 & 0 & 0 & $5.00 \mathrm{e} 3$ & $5.88 \mathrm{e} 3$ & $2.29 \mathrm{e} 7$ & $2.32 \mathrm{e} 7$ & $5.83 \mathrm{e} 7$ \\
$r=1 \mathrm{e}-1$ & 0 & 0 & 0 & $5.00 \mathrm{e} 5$ & $5.87 \mathrm{e} 5$ & $2.29 \mathrm{e} 7$ & $2.32 \mathrm{e} 7$ & $5.83 \mathrm{e} 7$ \\
$r=0.2$ & 0 & 0 & 0 & $2.00 \mathrm{e} 6$ & $2.34 \mathrm{e} 6$ & $2.31 \mathrm{e} 7$ & $2.36 \mathrm{e} 7$ & $5.84 \mathrm{e} 7$ \\
$r=0.3$ & 0 & 0 & 0 & $4.47 \mathrm{e} 6$ & $5.22 \mathrm{e} 6$ & $2.34 \mathrm{e} 7$ & $2.37 \mathrm{e} 7$ & $5.86 \mathrm{e} 7$ \\
$r=0.4$ & 0 & 0 & 0 & $7.84 \mathrm{e} 6$ & $9.09 \mathrm{e} 6$ & $2.40 \mathrm{e} 7$ & $2.43 \mathrm{e} 7$ & $5.90 \mathrm{e} 7$ \\
$r=0.57735$ & 0 & 0 & 0 & $1.47 \mathrm{e} 7$ & $1.66 \mathrm{e} 7$ & $2.75 \mathrm{e} 7$ & $2.78 \mathrm{e} 7$ & $6.03 \mathrm{e} 7$ \\
$r=0.7$ & 0 & 0 & 0 & $1.79 \mathrm{e} 7$ & $1.94 \mathrm{e} 7$ & $3.37 \mathrm{e} 7$ & $3.55 \mathrm{e} 7$ & $6.26 \mathrm{e} 7$ \\
$r=1$ & 0 & 0 & 0 & $2.01 \mathrm{e} 7$ & $2.10 \mathrm{e} 7$ & $5.00 \mathrm{e} 7$ & $7.05 \mathrm{e} 7$ & $9.11 \mathrm{e} 7$ \\
\hline \hline
\end{tabular}


Table 2: Effect of integartion points on the prediction of Eigenvalue

\begin{tabular}{ccccccccc}
\hline \hline $\begin{array}{c}\text { Integration } \\
\text { points }\end{array}$ & 1 & 2 & 3 & 4 & 5 & 6 & 7 & 8 \\
\hline$r=0$ & 0 & 0 & 1 & 1 & 1 & $1.03 \mathrm{e} 7$ & $1.22 \mathrm{e} 7$ & $3.23 \mathrm{e} 7$ \\
$r=1 \mathrm{e}-5$ & 0 & 0 & 1 & 1 & 1 & $1.03 \mathrm{e} 7$ & $1.22 \mathrm{e} 7$ & $3.23 \mathrm{e} 7$ \\
$r=1 \mathrm{e}-4$ & 0 & 0 & 1 & 1 & 1 & $1.03 \mathrm{e} 7$ & $1.22 \mathrm{e} 7$ & $3.23 \mathrm{e} 7$ \\
$r=1 \mathrm{e}-3$ & 0 & 0 & 1 & 1 & 7.23 & $1.03 \mathrm{e} 7$ & $1.22 \mathrm{e} 7$ & $3.23 \mathrm{e} 7$ \\
$r=1 \mathrm{e}-2$ & 0 & 0 & 1 & 1 & $7.23 \mathrm{e} 2$ & $1.03 \mathrm{e} 7$ & $1.22 \mathrm{e} 7$ & $3.23 \mathrm{e} 7$ \\
$r=1 \mathrm{e}-1$ & 0 & 0 & 1 & 1 & $7.17 \mathrm{e} 4$ & $1.04 \mathrm{e} 7$ & $1.23 \mathrm{e} 7$ & $3.25 \mathrm{e} 7$ \\
$r=0.2$ & 0 & 0 & 1 & 1 & $2.79 \mathrm{e} 5$ & $1.05 \mathrm{e} 7$ & $1.26 \mathrm{e} 7$ & $3.30 \mathrm{e} 7$ \\
$r=0.3$ & 0 & 0 & 1 & 1 & $6.02 \mathrm{e} 5$ & $1.08 \mathrm{e} 7$ & $1.32 \mathrm{e} 7$ & $3.39 \mathrm{e} 7$ \\
$r=0.4$ & 0 & 0 & 1 & 1 & $1.01 \mathrm{e} 6$ & $1.12 \mathrm{e} 7$ & $1.40 \mathrm{e} 7$ & $3.51 \mathrm{e} 7$ \\
$r=0.57735$ & 0 & 0 & 1 & 1 & $1.80 \mathrm{e} 6$ & $1.21 \mathrm{e} 7$ & $1.62 \mathrm{e} 7$ & $3.83 \mathrm{e} 7$ \\
$r=0.7$ & 0 & 0 & 1 & 1 & $2.32 \mathrm{e} 6$ & $1.27 \mathrm{e} 7$ & $1.84 \mathrm{e} 7$ & $4.18 \mathrm{e} 7$ \\
$r=1$ & 0 & 0 & 1 & 1 & $3.29 \mathrm{e} 6$ & $1.39 \mathrm{e} 7$ & $2.66 \mathrm{e} 7$ & $5.35 \mathrm{e} 7$ \\
\hline \hline
\end{tabular}


Table 3: Effect of integartion points on the prediction of Eigen value $(v=0.499)$

\begin{tabular}{ccccccccc}
\hline \hline $\begin{array}{c}\text { Integration } \\
\text { points }\end{array}$ & 1 & 2 & 3 & 4 & 5 & 6 & 7 & 8 \\
\hline$r=0$ & 0 & 0 & 0 & 0 & 0 & $1.99 \mathrm{e} 7$ & $2.01 \mathrm{e} 7$ & $1.00 \mathrm{e} 10$ \\
$r=(0.5-v) \mathrm{e}-1$ & 0 & 0 & 0 & $4.65 \mathrm{e} 1$ & $5.89 \mathrm{e} 1$ & $1.99 \mathrm{e} 7$ & $2.01 \mathrm{e} 7$ & $1.00 \mathrm{e} 10$ \\
$r=(0.5-v)$ & 0 & 0 & 0 & $4.65 \mathrm{e} 3$ & $5.89 \mathrm{e} 3$ & $1.99 \mathrm{e} 7$ & $2.01 \mathrm{e} 7$ & $1.00 \mathrm{e} 10$ \\
$r=(0.5-v) \mathrm{e} 1$ & 0 & 0 & 0 & $4.64 \mathrm{e} 5$ & $5.88 \mathrm{e} 5$ & $1.99 \mathrm{e} 7$ & $2.01 \mathrm{e} 7$ & $1.00 \mathrm{e} 10$ \\
$r=0.57735$ & 0 & 0 & 0 & $1.84 \mathrm{e} 7$ & $1.87 \mathrm{e} 7$ & $1.84 \mathrm{e} 9$ & $2.12 \mathrm{e} 9$ & $1.02 \mathrm{e} 10$ \\
$r=1$ & 0 & 0 & 0 & $1.84 \mathrm{e} 7$ & $1.88 \mathrm{e} 7$ & $6.13 \mathrm{e} 9$ & $6.64 \mathrm{e} 9$ & $1.16 \mathrm{e} 10$ \\
\hline \hline
\end{tabular}

Table 4: Effect of integartion points on the prediction of Eigen value $(v=0.4995)$

\begin{tabular}{ccccccccc}
\hline \hline $\begin{array}{c}\text { Integration } \\
\text { points }\end{array}$ & 1 & 2 & 3 & 4 & 5 & 6 & 7 & 8 \\
\hline$r=0$ & 0 & 0 & 0 & 0 & 0 & $1.99 \mathrm{e} 7$ & $2.01 \mathrm{e} 7$ & $2.01 \mathrm{e} 10$ \\
$r=(0.5-v) \mathrm{e}-1$ & 0 & 0 & 0 & $2.31 \mathrm{e} 1$ & $2.94 \mathrm{e} 1$ & $1.99 \mathrm{e} 7$ & $2.01 \mathrm{e} 7$ & $2.01 \mathrm{e} 10$ \\
$r=(0.5-v)$ & 0 & 0 & 0 & $2.32 \mathrm{e} 3$ & $2.94 \mathrm{e} 3$ & $1.99 \mathrm{e} 7$ & $2.01 \mathrm{e} 7$ & $2.01 \mathrm{e} 10$ \\
$r=(0.5-v) \mathrm{e} 1$ & 0 & 0 & 0 & $2.32 \mathrm{e} 5$ & $2.94 \mathrm{e} 5$ & $1.99 \mathrm{e} 7$ & $2.01 \mathrm{e} 7$ & $2.01 \mathrm{e} 10$ \\
$r=0.57735$ & 0 & 0 & 0 & $1.84 \mathrm{e} 7$ & $1.87 \mathrm{e} 7$ & $3.67 \mathrm{e} 9$ & $4.24 \mathrm{e} 9$ & $2.04 \mathrm{e} 10$ \\
$r=1$ & 0 & 0 & 0 & $1.84 \mathrm{e} 7$ & $1.87 \mathrm{e} 7$ & $1.22 \mathrm{e} 10$ & $1.33 \mathrm{e} 10$ & $2.33 \mathrm{e} 10$ \\
\hline \hline
\end{tabular}

Table 5: Effect of integartion points on the prediction of Eigen value $(v=0.49995)$

\begin{tabular}{ccccccccc}
\hline \hline $\begin{array}{c}\text { Integration } \\
\text { points }\end{array}$ & 1 & 2 & 3 & 4 & 5 & 6 & 7 & 8 \\
\hline$r=0$ & 0 & 0 & 0 & 0 & 0 & $1.99 \mathrm{e} 7$ & $2.01 \mathrm{e} 7$ & $2.01 \mathrm{e} 11$ \\
$r=(0.5-v) \mathrm{e}-1$ & 0 & 0 & 0 & $2.31 \mathrm{e} 0$ & $2.93 \mathrm{e} 0$ & $1.99 \mathrm{e} 7$ & $2.01 \mathrm{e} 7$ & $2.01 \mathrm{e} 11$ \\
$r=(0.5-v)$ & 0 & 0 & 0 & $2.31 \mathrm{e} 2$ & $2.93 \mathrm{e} 2$ & $1.99 \mathrm{e} 7$ & $2.01 \mathrm{e} 7$ & $2.01 \mathrm{e} 11$ \\
$r=(0.5-v) \mathrm{e} 1$ & 0 & 0 & 0 & $2.31 \mathrm{e} 4$ & $2.93 \mathrm{e} 4$ & $1.99 \mathrm{e} 7$ & $2.01 \mathrm{e} 7$ & $2.01 \mathrm{e} 11$ \\
$r=0.57735$ & 0 & 0 & 0 & $1.84 \mathrm{e} 7$ & $1.87 \mathrm{e} 7$ & $3.67 \mathrm{e} 10$ & $4.23 \mathrm{e} 10$ & $2.04 \mathrm{e} 11$ \\
$r=1$ & 0 & 0 & 0 & $1.84 \mathrm{e} 7$ & $1.87 \mathrm{e} 7$ & $1.22 \mathrm{e} 13$ & $1.32 \mathrm{e} 11$ & $2.33 \mathrm{e} 11$ \\
\hline \hline
\end{tabular}


Table 6: Effect of integartion points on the prediction of Eigen value $(v=0.499995)$

\begin{tabular}{ccccccccc}
\hline $\begin{array}{c}\text { Integration } \\
\text { points }\end{array}$ & 1 & 2 & 3 & 4 & 5 & 6 & 7 & 8 \\
\hline$r=0$ & 0 & 0 & 0 & 0 & 0 & $1.99 \mathrm{e} 7$ & $2.01 \mathrm{e} 7$ & $2.01 \mathrm{e} 13$ \\
$r=(0.5-v) \mathrm{e}-1$ & 0 & 0 & 0 & $2.31 \mathrm{e}-1$ & $2.93 \mathrm{e}-1$ & $1.99 \mathrm{e} 7$ & $2.01 \mathrm{e} 7$ & $2.01 \mathrm{e} 13$ \\
$r=(0.5-v)$ & 0 & 0 & 0 & $2.31 \mathrm{e} 1$ & $2.93 \mathrm{e} 1$ & $1.99 \mathrm{e} 7$ & $2.01 \mathrm{e} 7$ & $2.01 \mathrm{e} 13$ \\
$r=(0.5-v) \mathrm{e} 1$ & 0 & 0 & 0 & $2.31 \mathrm{e} 3$ & $2.93 \mathrm{e} 3$ & $1.99 \mathrm{e} 7$ & $2.01 \mathrm{e} 7$ & $2.01 \mathrm{e} 13$ \\
$r=0.57735$ & 0 & 0 & 0 & $1.84 \mathrm{e} 7$ & $1.87 \mathrm{e} 7$ & $3.67 \mathrm{e} 11$ & $4.22 \mathrm{e} 11$ & $2.04 \mathrm{e} 12$ \\
$r=1$ & 0 & 0 & 0 & $1.84 \mathrm{e} 7$ & $1.87 \mathrm{e} 7$ & $1.22 \mathrm{e} 13$ & $1.32 \mathrm{e} 12$ & $2.33 \mathrm{e} 12$ \\
\hline \hline
\end{tabular}

Table 7: Effect of integartion points on the prediction of Eigen value $(v=0.4999995)$

\begin{tabular}{ccccccccc}
\hline $\begin{array}{c}\text { Integration } \\
\text { points }\end{array}$ & 1 & 2 & 3 & 4 & 5 & 6 & 7 & 8 \\
\hline$r=0$ & 0 & 0 & 0 & 0 & 0 & $1.99 \mathrm{e} 7$ & $2.01 \mathrm{e} 7$ & $2.01 \mathrm{e} 9$ \\
$r=(0.5-v) \mathrm{e}-1$ & 0 & 0 & 0 & $2.33 \mathrm{e}-2$ & $2.92 \mathrm{e}-2$ & $1.99 \mathrm{e} 7$ & $2.01 \mathrm{e} 7$ & $2.01 \mathrm{e} 13$ \\
$r=(0.5-v)$ & 0 & 0 & 0 & $2.32 \mathrm{e} 0$ & $2.94 \mathrm{e} 0$ & $1.99 \mathrm{e} 7$ & $2.01 \mathrm{e} 7$ & $2.01 \mathrm{e} 13$ \\
$r=(0.5-v) \mathrm{e} 1$ & 0 & 0 & 0 & $2.32 \mathrm{e} 2$ & $2.93 \mathrm{e} 2$ & $1.99 \mathrm{e} 7$ & $2.01 \mathrm{e} 7$ & $2.01 \mathrm{e} 13$ \\
$r=0.57735$ & 0 & 0 & 0 & $1.84 \mathrm{e} 7$ & $1.87 \mathrm{e} 7$ & $3.66 \mathrm{e} 12$ & $4.22 \mathrm{e} 12$ & $2.04 \mathrm{e} 13$ \\
$r=1$ & 0 & 0 & 0 & $1.84 \mathrm{e} 7$ & $1.87 \mathrm{e} 7$ & $1.22 \mathrm{e} 13$ & $1.32 \mathrm{e} 13$ & $2.33 \mathrm{e} 13$ \\
\hline \hline
\end{tabular}

Table 8: Condition number for various Poisson's ratio

\begin{tabular}{ccccccc}
\hline \hline \multirow{2}{*}{$\begin{array}{c}\text { Poisson's } \\
\text { ratio }\end{array}$} & $r=0$ & $r=(0.5-v) \mathrm{e}-1$ & $r=(0.5-v)$ & $r=(0.5-v) \mathrm{e} 1$ & $r=0.57735$ & $r=1$ \\
\hline$v=0.499$ & inf & $2.22 \mathrm{E}+17$ & $3.16 \mathrm{E}+17$ & $4.46 \mathrm{E}+17$ & $9.55 \mathrm{E}+16$ & $1.88 \mathrm{E}+18$ \\
$v=0.4995$ & inf & $2.84 \mathrm{E}+17$ & $3.48 \mathrm{E}+17$ & $2.62 \mathrm{E}+17$ & $9.23 \mathrm{E}+17$ & $1.34 \mathrm{E}+17$ \\
$v=0.49995$ & inf & $3.26 \mathrm{E}+17$ & $2.56 \mathrm{E}+17$ & $3.72 \mathrm{E}+17$ & $6.01 \mathrm{E}+17$ & $2.50 \mathrm{E}+17$ \\
$v=0.499995$ & inf & $1.39 \mathrm{E}+18$ & $3.46 \mathrm{E}+17$ & $1.13 \mathrm{E}+19$ & $1.75 \mathrm{E}+18$ & $1.61 \mathrm{E}+17$ \\
$v=0.499995$ & inf & $4.94 \mathrm{E}+17$ & $1.32 \mathrm{E}+17$ & $2.42 \mathrm{E}+17$ & $3.35 \mathrm{E}+17$ & $3.23 \mathrm{E}+17$ \\
\hline \hline
\end{tabular}

